\title{
SDN and Virtualization-Based LTE Mobile Network Architectures: A Comprehensive Survey
}

\author{
Van-Giang Nguyen ${ }^{1} \cdot$ Truong-Xuan $\mathrm{Do}^{1} \cdot$ \\ YoungHan Kim ${ }^{1}$
}

Published online: 6 August 2015

(C) The Author(s) 2015. This article is published with open access at Springerlink.com

\begin{abstract}
Software-defined networking (SDN) features the decoupling of the control plane and data plane, a programmable network and virtualization, which enables network infrastructure sharing and the "softwarization" of the network functions. Recently, many research works have tried to redesign the traditional mobile network using two of these concepts in order to deal with the challenges faced by mobile operators, such as the rapid growth of mobile traffic and new services. In this paper, we first provide an overview of SDN, network virtualization, and network function virtualization, and then describe the current LTE mobile network architecture as well as its challenges and issues. By analyzing and categorizing a wide range of the latest research works on SDN and virtualization in LTE mobile networks, we present a general architecture for SDN and virtualization in mobile networks (called SDVMN) and then propose a hierarchical taxonomy based on the different levels of the carrier network. We also present an in-depth analysis about changes related to protocol operation and architecture when adopting SDN and virtualization in mobile networks. In addition, we list specific use cases and applications that benefit from SDVMN. Last but not least, we discuss the open issues and future research directions of SDVMN.
\end{abstract}

Keywords Software defined networking - Network virtualization - Network function virtualization · Future mobile network - LTE - Evolved packet core $\cdot 5 \mathrm{G}$ mobile network

YoungHan Kim

younghak@ssu.ac.kr; yhkim@dcn.ssu.ac.kr

Van-Giang Nguyen

nvgiang@dcn.ssu.ac.kr

Truong-Xuan Do

xuan@dcn.ssu.ac.kr

1 School of Electronic Engineering, Soongsil University, Seoul, South Korea 


\section{Introduction}

Recently, the explosion of handheld devices has caused an exponential increase in mobile data traffic usage. Cisco forecasts [1] have shown that mobile data traffic will grow by $61 \%$ from 2013 to 2018. This growth is based on current mobile data services and applications, such as mobile games, mobile videos, and location-based check-in services. This trend is expected to keep growing due to the appearance of new mobile services and applications. The significant growth in mobile traffic, new network services, and applications are pushing carrier network operators to upgrade their systems in order to meet new requirements and increasing customer demands. This imposes challenges for the current cellular network architecture, such as high upgrade costs, complex operation, and slow deployment of new innovations and services [2]. In addition, the simultaneous appearance of multiple wireless technologies has also brought challenges, including the interworking between these diverse technologies, inter-cell interference, and radio resource management in the dense network environment.

However, the current mobile network has several issues regarding its inherent design; thus, the afore-mentioned challenges cannot easily be addressed without radically changing the architecture. First, the current implementations of mobile networks are very expensive and difficult to modify. For example, the current mobile core networks rely on proprietary hardware appliances [3], which make it difficult to upgrade them as they become obsolete. Second, the tightly coupling between the control plane and data plane makes the current mobile network very slow and complicated when new network services or innovations need to be implemented. The manual configuration for each device also brings burdens for network operation and maintenance. Last, the current mobile network manages network resources inefficiently. Traditionally, radio resource management is performed in a distributed fashion [2], where each base station has its own decision on radio resources. This makes radio resource and inter-cell interference management highly complex and suboptimal.

The software-defined network (SDN) [4], a new network paradigm, came from the campus and enterprise network environments with the invention of the Openflow concept [5]. The main concepts of SDN include the separation of the control data plane, and a programmable network. SDN facilitates network configuration and management by pushing all control tasks to a centralized controller. Moreover, SDN speeds the deployment of new innovations or services and reduces operational costs through programmable interfaces (e.g., Openflow [6], ForCES [7], and PCEP [8]) in the controller. Due to the benefits of SDN, mobile network operators have recently realized that SDN is a promising solution for their current mobile networks.

Virtualization refers to two types of technologies: network virtualization [9] (NV) and network function virtualization (NFV) [10]. NV allows multiple virtual networks to operate on the same network infrastructure. This enables the coexistence of multiple service providers or carrier network operators in the shared infrastructure. NFV refers to the implementation of network functions in software running on general purpose computing/storage platforms. In the mobile network, with the migration from hardware to software appliances, NFV is expected to lower not only equipment costs (CAPEX) but also the operational costs (OPEX).

SDN and virtualization are two major trends in the evolution of the mobile network. Not long ago, the fifth generation (5G) cellular network was defined and discussed globally. The 5G-PPP group [11], which consists of some large projects in Europe such as METIS 
[12], iJOIN [13], Mobile Cloud Networking [14], and CROWD [15], has released its plan and perspectives for future mobile networks until 2020. It considers SDN and NFV to be some of the key technologies for the $5 \mathrm{G}$ cellular network architecture. For SDN and NFV, some of the top benefits being focused on include satisfying the need for network operators to speed up their service innovations and simplifying network management.

In this paper, we present a comprehensive survey about the impact of SDN and virtualization on mobile network architecture. To the best of our knowledge, this is the first comprehensive and profound survey on SDN and virtualization in the mobile network architecture. We found few papers surveying this topic. In [16], the authors briefly presented a survey on SDN concepts and its benefits while considering a limited number of surveyed works. In [17], the authors considered two main streams: SDN-based mobile network and wireless network virtualization, as well as the simple integration of these two approaches. The section focusing on virtualization only covered network virtualization in a general wireless network architecture, and did not cover both NV and NFV in wireless mobile environments. The number of surveyed papers was also limited. In our paper, we aim at providing a more comprehensive and general survey about SDN and virtualization in the mobile network by covering and categorizing a wide range of the latest research works. The main contributions of this paper are as follows:

- Cover a wide range of up-to-date research works on SDN and virtualization in mobile network.

- Present a general architecture for SDN and virtualization in the mobile network called SDVMN.

- Describe the benefits that these two concepts bring to each level of the mobile cellular network.

- Propose a hierarchical taxonomy which identifies main issues in the different levels of carrier network and figure out main groups of SDN and virtualization-based solutions to address these issues.

- Analyze in-depth how SDN and virtualization can change the protocol operation and architecture of the current carrier network.

- Provide a list of specific use cases and applications that can benefit from SDVMN.

The remainder of the paper is organized as follows. In Sect. 2, we review the current mobile network architecture and its challenges and issues, and then briefly present the concepts of SDN, NV, and NFV. In Sect. 3, we describe a general architecture for SDN and virtualization in the mobile network, as well as its benefits. The taxonomy tree of our survey is described in Sect. 4. Section 5 surveys current approaches to SDVMN organized according to the proposed taxonomy. We discuss open issues and future research directions in Sect. 6. Finally, we conclude our survey in Sect. 7.

\section{Background}

This section describes the mobile network architecture and its problems and issues. Then, we review the key technologies, including software-defined networking, network virtualization, and network function virtualization. 


\subsection{Long Term Evolution (LTE) System}

Up to now, the mobile network has gone through many generations during its evolution, such as GPRS and UMTS. In this paper, we only focus on LTE, the latest mobile network generation.

The LTE network is divided into two parts: the LTE part deals with radio access technologies, while the evolved packet core (EPC) deals with the technology related to a core network. User equipment (UE) connects to an eNodeB via radio interface. The eNodeB performs radio resource management functions such as allocating radio resources and managing inter-cell interference. The eNodeB connects to a serving gateway (SGW) through a mobile backhaul network. The SGW serves a large number of eNodeBs and acts as the local mobility anchor point for the inter-eNodeB handover. The SGW is connected to a packet data network (PDN) gateway (PGW), which performs UE IP address allocation, policy enforcement, packet filtering and charging. It is a termination point for the packet data interface towards the external network. The mobility management entity (MME) is the main control entity and is responsible for maintaining mobility management states for UEs and setting up bearers to carry user traffic. The home subscriber server (HSS) is a central database where user profiles are stored. It is in charge of UE authentication and authorization. The policy and charging rules function (PCRF) provides QoS and charging rules to the PGW. User data packets are forwarded through GTP (GPRS tunneling protocol) tunnels between the eNodeB and PGW. Figure 1 depicts the overall architecture of mobile network.

Although the LTE came out to cope with the increasing growth of mobile devices, it still has many issues and challenges. In radio access and the mobile backhaul network, the deployment of many base stations and the distribution of the control plane, the LTE is hard to manage, which results in inefficient radio resource management. It is also hard for the network mobile operator to deal with the interference problem between cells or base stations. In the mobile core network, having no clear separation between the control and data planes makes the network difficult to manage and control. The use of proprietary hardware appliances for network entities results in high deployment and operational costs and inefficient resource utilization, which slows down the time-to-market for new innovations and impacts the operators' revenue.

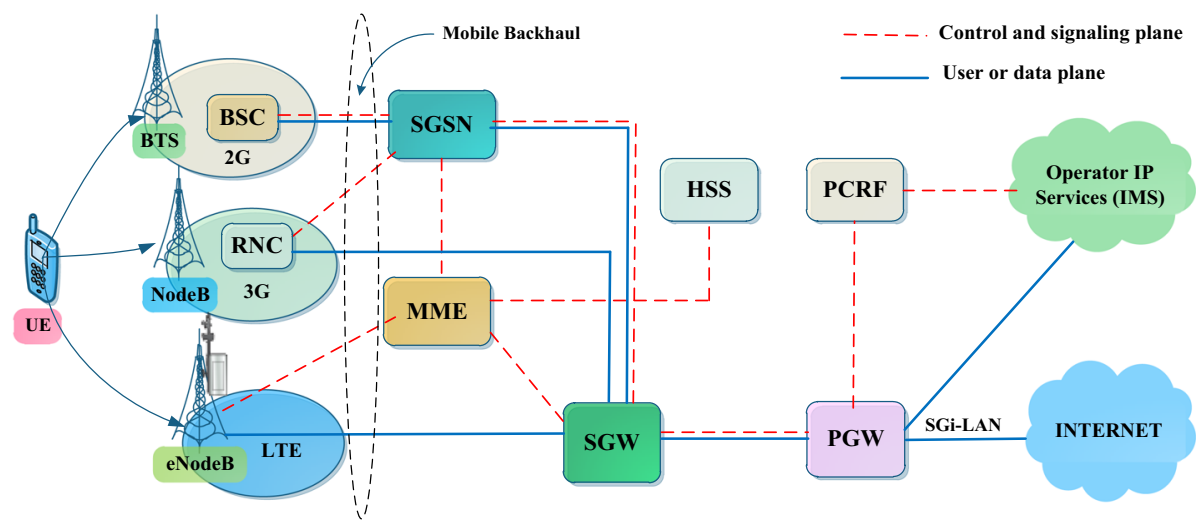

Fig. 1 Architecture of LTE mobile network [18] 


\subsection{Software-Defined Networking}

SDN is a centralized paradigm in which the network intelligence or control plane is lifted up to a logically centralized entity, or SDN controller. The data plane consists of simple forwarding devices that are controlled by the SDN controller through programmable interfaces. Nowadays, Openflow [6] is a widely used SDN interface that is maintained by the Open Networking Foundation (ONF) [19]. There have been several approaches for SDN, as surveyed in [20-22]. Figure 2 depicts a typical architecture of SDN. The SDN architecture consists of three main layers: the infrastructure layer, control layer, and application layer. The infrastructure layer or data plane is composed of forwarding devices, such as virtual switches and physical switches. The control layer or control plane comprises a set of SDN controllers (e.g., a Floodlight controller [23] or OpenDaylight controller [24]) that provide control tasks through southbound interfaces (e.g., Openflow, ForCES and PCEP). These controllers communicate with others using east/westbound interfaces (e.g. SDNi interfaces [25]). The application layer or application plane consists of one or more applications, such as routing, security application and monitoring applications. The SDN applications communicate their network requirements towards the controllers via northbound interfaces such as REST API or Java API. The main benefits of SDN include ease of configuration and management, high rate of innovation, and network programmability

\subsection{Network Virtualization (NV)}

As surveyed in [9], network virtualization allows multiple service providers to compose their own virtual networks that coexist together on the shared physical infrastructure. Service providers can deploy and manage customized end-to-end services on those virtual networks effectively for the end users. Historically, virtual local network (VLAN) and virtual private network (VPN) have been two popular technologies for creating logical

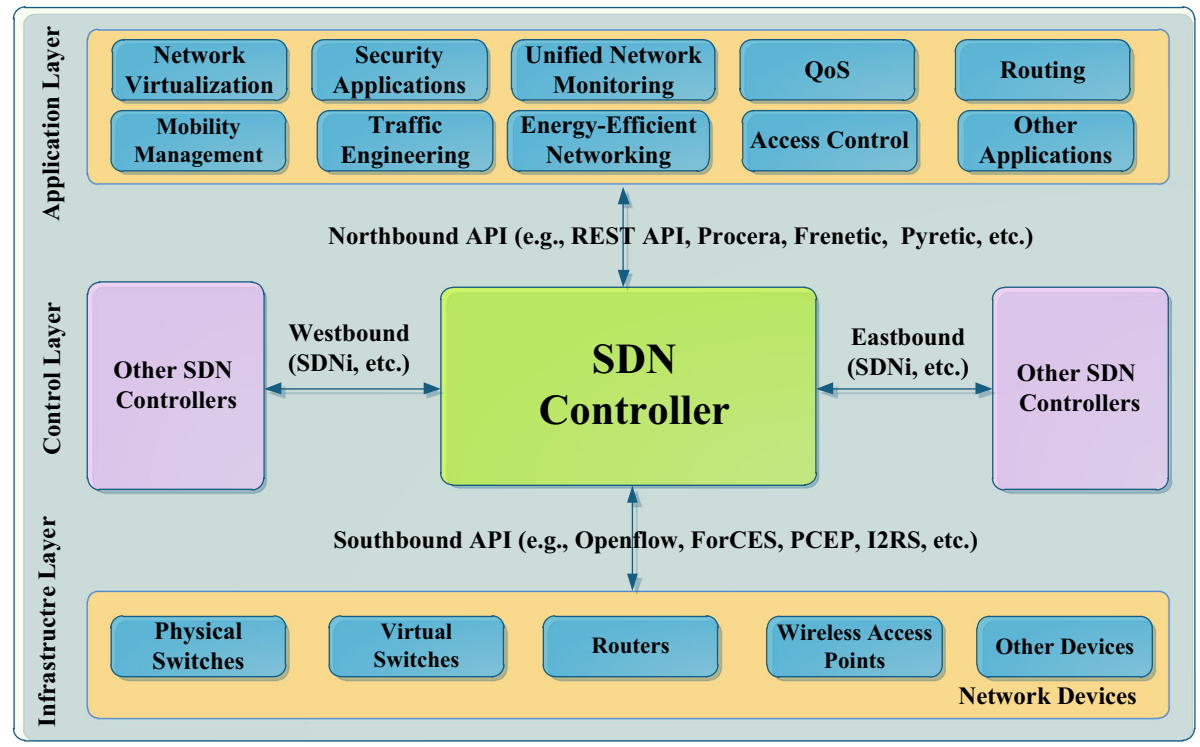

Fig. 2 Overview of SDN architecture [26] 
networks on physical substrate. Before the advent of SDN and Openflow, among projects working in the area of NV, we may cite some important projects such as Planet Lab [27], 4WARD [28], GENI [29], and VINI [30]. In the Openflow network, virtual networks are created by some special controllers, such as FlowVisor [31] and OpenVirteX [32]. FlowVisor and OpenVirteX allow the underlying Openflow-based physical network to be sliced into multiple isolated virtual networks or slices and control of each slice to be delegated to a specific controller, as shown in Fig. 3a. The main benefits of NV include the maximization of resource usage, the reduction of equipment and management costs, and QoS improvement, thus encouraging network innovation and the deployment of distinct network services.

\subsection{Network Function Virtualization (NFV)}

NFV refers to the virtualization of network functions and their migration from stand-alone boxes based on dedicated hardware to software appliances running on a cloud computing system or an IT standard infrastructure [10]. NFV is promoted and maintained by the European Telecommunications Standards Institute (ETSI) [33]. NFV provides many benefits to network operators, including equipment cost reduction, fast time to market, a wide variety of eco-systems and the encouragement of openness, multi-tenancy support, energy consumption reduction, and scalability [34]. ETSI NFV introduced some use cases of NFV in the mobile network environment [3]. The reference NFV architecture is depicted in Fig. 3b.

In summary, the SDN, NV and NFV technologies aim at accelerating a software-based approach to make networking more scalable, flexible, efficient and innovative. These technologies are all complementary approaches to each other.

\section{SDN and Virtualization-Based Mobile Network (SDVMN)}

In this section, we describe a generic model of SDVMN architecture. By generalizing the architecture for a mobile network based on SDN and virtualization, we aim at providing the big picture of SDVMN, how the SDN and virtualization can change the current mobile network, and which parts of the mobile network are currently being tackled by researchers

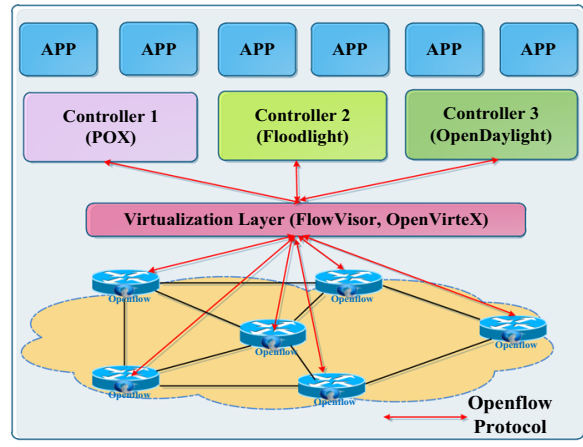

(a)

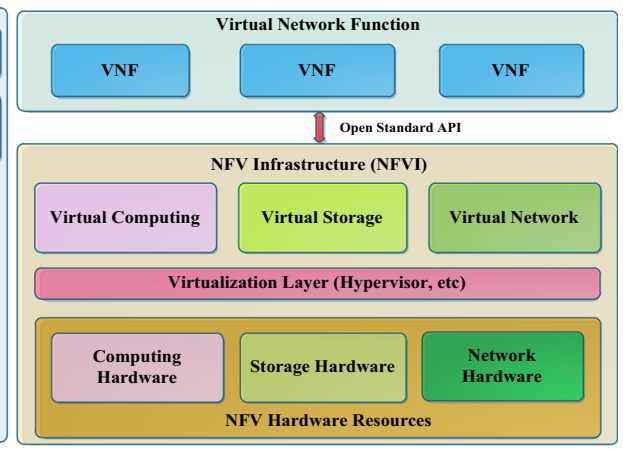

(b)

Fig. 3 a Example of network virtualization in an Openflow network and b Reference NFV architecture [35] 
in academia and industry. In addition, we also analyze and discuss the benefits we can get from SDVMN.

\subsection{SDVMN Architecture}

Figure 4 depicts the overall architecture of a future mobile network that has realized SDN and virtualization technology. The key features of the SDVMN architecture are the separation of the control and the data plane and the virtualization of network infrastructures, as well as the virtualization of network functions. To make the architecture more easily understandable, we will present the detailed architecture of SDVMN in two directions: as a vertical description from bottom to top and then in a horizontal description from left to right.

Vertically, we follow the reference model of the current 3GPP evolution packet system, which consists of three main parts: a radio access network (RAN), mobile backhaul, and the mobile packet core network. The UE uses the services from external networks (IMS system, the Internet, etc.) through a SGi-LAN interface, as defined in [18], where a set of middleboxes are deployed. Horizontally, we show four planes of SDN architectures: the data plane, control plane, application plane and management plane. One of our main objectives is to figure out a generic architectural model for SDVMN and map it into the SDN reference architecture. By using this generic model, we can easily understand how SDN technology and virtualization are being used in mobile networks and how our hierarchical taxonomy is made according to this model.

As depicted in Fig. 4, the RAN part is the heterogeneous access network environment which includes different access technologies such as GSM, UMTS, LTE and Wi-Fi. These radio access networks can be programmable and under the supervision of a pool of SDNRAN controllers. The second part of the SDVMN is the backhaul network which connects a radio access network and a mobile core network. MPLS or optical technologies are two technologies currently used in mobile backhaul networks. Within the SDN concept, the network equipment used in MPLS or optical networks is enhanced with programmability. These network equipment can be dedicated hardware-based and programmable switches,
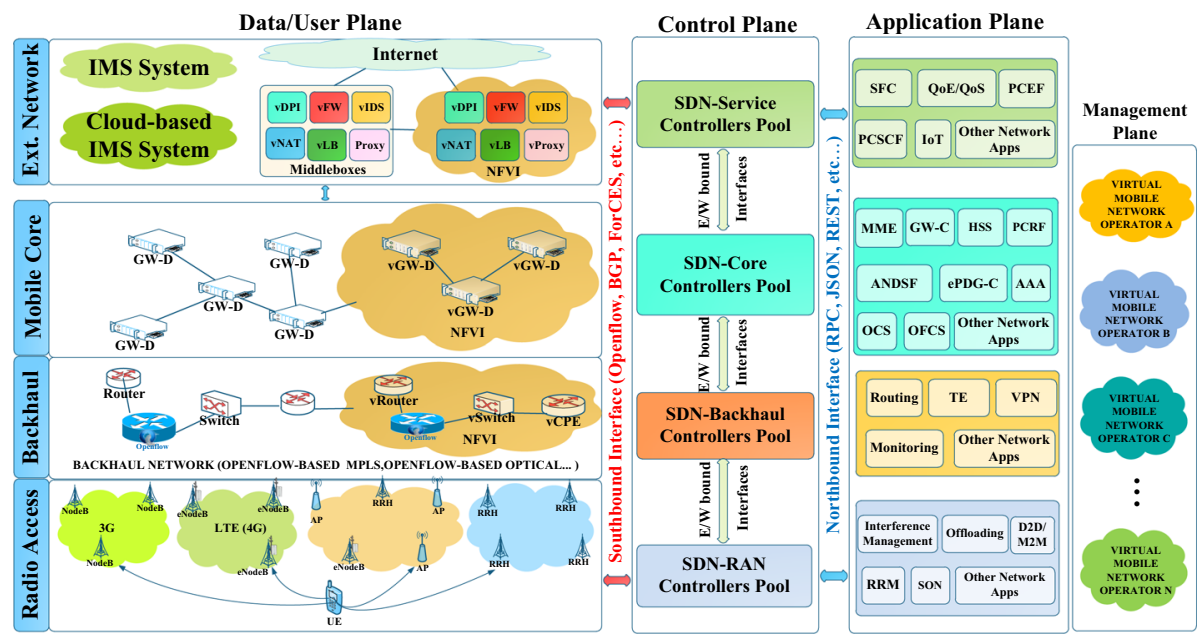

Fig. 4 General architecture of a SDN and virtualization-based mobile network (SDVMN) 
routers or virtualized switches that are realized as virtual machines (VMs) running on a cloud computing system or NFVI. The underlying infrastructure of the mobile backhaul network is controlled by a pool of SDN-backhaul controllers.

The third part of the SDVMN is the mobile core network. In contrast to the traditional mobile core networks, the SDVMN core network is composed of simple network gateways called GW-Ds, which can either be dedicated hardware servers or software appliances running on the NFVI environment. The SDVMN core network can also be programmed with SDN-core controllers through open APIs. GW-Ds act as an anchor point for intra-/ inter-handover in the mobile network, and are a point of connection to external networks (e.g., IMS, the Internet, etc.). By using distributed GW-Ds, the SDVMN can eliminate the single point of failure issue, provide scalability and guarantee service availability for users.

Last part is the external network placed on the top of the SDVMN architecture. The external network is an IMS system or Internet, which provides the services for UEs. Before flowing to the Internet, the user data traffic needs to pass through a set of middleboxes placed behind the SGi-LAN interface. These middleboxes help ensure security, optimize performance, and facilitate remote access. Some examples of middleboxes include firewalls, load balancers, and WAN optimization. By realizing the concept of NFV, these middleboxes can be deployed as software appliances running on an NFVI environment. For IMS systems, with the emergence of NFV, traditional IMS is being cloudified, with the migration of proprietary hardware-based systems within a dedicated network infrastructure into software-based deployments in a cloud infrastructure.

Now, let's look into the horizontal side of SDVMN architecture. Four layers of the general SDN architecture are separated explicitly in Fig. 4. The first layer (data or user plane) enables user data traffic to be delivered through the RAN to the external network. The second layer is the control layer, where a pool of SDN controllers can be deployed for every part of the SDVMN. In fact, we can assume that the entire SDVMN architecture can be controlled and managed by a super-SDN controller. However, this makes the architecture too difficult and complex to grasp fully. Therefore, we divide this super-SDN controller into four controller levels, according to the 4-tier model of mobile networks, in order to ease comprehension: the SDN-RAN controller, the SDN-Backhaul controller, the SDN-Core controller, and the SDN-Service controller. From a global view of the network state information, these SDN controllers can perform many actions to control and manage the RAN, the mobile backhaul, the mobile packet core, and the external network, respectively. For example, the SDN-RAN controller is responsible for controlling and managing the radio resources. Our use of the term "pool" implies that we can deploy the SDN controller in a distributed manner with a set of collaborated controllers to deal with the single point of failure issue in the control layer. These controllers cooperate with each other through east/westbound interfaces. Similar to the network devices in the data plane, the SDN controllers can also be deployed as either hardware-based or software-based controllers running in an NFVI environment.

The next layer is the application layer or application plane. Throughout RAN to the external network, the application layer consists of a series of network control functions placed on the top of the SDN controllers. Corresponding to the RAN part, the network control functions can include interference management, radio resource management and offloading. Corresponding to backhaul network part, functions which can be pushed into the controller include backhaul resource management, traffic engineering, and monitoring. Corresponding to the core network part, all network control functions including MME, GW-C, PCRF, HSS and authentication systems (AAA) can be packaged as applications running on SDN-core controllers or VMs running on an NFVI environment. 
Corresponding to the external part, network functions can be deployed as a chain. Service function chaining (SFC) and network service chaining (NSC) [36] are two typical examples of applications for this part, which refers to a carrier-grade process for the continuous delivery of services based on network function associations.

Last layer at the horizontal side of the SDVMN architectural model is the management layer or management plane. As defined in [37], the management plane is considered to be another plane in SDN architecture, which covers static tasks that are better handled outside the application, control and data planes and refers to human-centric interaction. In the SDVMN architecture, the term "management plane" refers to the operator's management. The network virtualization technology allows multiple network operators to share the same underlying SDVMN infrastructure. In other words, the underlying SDVMN infrastructure can be divided into several virtual networks, from the RAN to the core part, according to the customized requirements of various mobile virtual network operators (MVNO).

In order to operate the SDVMN architecture smoothly, the standard interfaces are needed to define between layers. The southbound interface is used by the SDN controllers to program the mobile network data plane according to various policies and requests from the virtual operators. The southbound interface candidates include Openflow, ForCES, PCEP and BGP. The northbound interface [38] is a programmatic interface that lives on the northern side of the controller. Typically, a northbound interface abstracts the low-level instruction sets used by the southbound interfaces to program forwarding devices. Some typical northbound interfaces considered in the SDVMN include the REST API, RPCJSON, and Java APIs. East/westbound interfaces are used to interconnect among controllers in order to achieve scalability in a multi-domain network. A typical east/westbound interface is SDNi, which is being implemented in the OpenDaylight controller [25]. In the SDVMN, the role of east/westbound interfaces is not only to deal with scalability problems but also to synchronize between parts in an SDVMN, so that it can provide a smooth endto-end connection for users.

\subsection{Benefits of SDVMN Architecture}

SDVMN architecture offers several useful features for the mobile network evolution: (a) network functions that are decoupled from specific hardware devices to software on the general computing platform; (b) a centralized control plane and the enabling of a programmable network; and (c) a physical network infrastructure that is sliced into virtual networks to enable network sharing. These features can be applied into every level of a mobile network architecture and result in many general key benefits, such as CAPEX and OPEX reduction, easy management and operation, speedy innovation deployment without interfering in-service networks, and efficient resource utilization. These benefits are specific to each level of mobile network architecture and are described below:

\subsubsection{Benefits in Radio Access Network}

By adopting the SDN and virtualization in the RAN part, we gain some following benefits. First, with network virtualization, the physical RAN resources (i.e. eNobeB) can be abstracted and sliced into virtual RAN resources and shared by multiple operators, so that the operators can save significantly on their deployment and operational costs. Through a SDN-RAN controller, the service providers can customize their own virtual network slices. Second, via the virtualization and centralization of RAN functions, these RAN functions can be shared by other radio functions. This improves resource utilization and 
throughput. Last, the radio resource management task is simplified by using a centralized controller for the RAN. The SDN-RAN controller is in charge of scheduling and allocating radio resources for radio access elements. The latest radio resource management and interference coordination algorithms can easily be upgraded and deployed on the SDNRAN controller. As a result, the SDN-RAN controller can fairly allocate radio resources for radio access elements and calculate an interference map to cancel or exploit interference between adjacent cells, thus improving RAN performance.

\subsubsection{Benefits in Mobile Backhaul Network}

The deployment of a mobile backhaul network is often costly. With the support of NFV and SDN, mobile backhaul network equipment can be enhanced with programmability or comprise virtualized appliances running on a commodity hardware platform, which is often cheaper than a dedicated hardware-based deployment. Slicing the mobile backhaul infrastructure provides the ability to share the network resource between different mobile operators. This enables traffic to be redirected from one operator to another in cases of congestion or heavy traffic conditions.

\subsubsection{Benefits in the Mobile Core Network}

In this core part, the main benefits come from the virtualization of core network functions, the programmability of the core network, the centralization of the control plane, and the virtual network operator concept. First, the virtualization of core network functions helps to reduce CAPEX and OPEX, supports multi-tenancy, and scales core network resources up and down rapidly, according to the demands of mobile operators. Second, the programmability of the core network simplifies its management, makes network configuration easier, and enables new innovations, with faster time to market. By using a centralized controller, the mobile network can control QoS in a fine-grained manner, according to various subscriber attributes and services' requirements. Last, slicing enables multiple virtual network operators to run on the same physical core network infrastructure, which optimizes the device cost and resource utilization.

\subsubsection{Benefits in the External Network}

Here, the main benefits come from the virtualization of IMS functions and the introduction of SFC. First, the virtualization of IMS functions on a cloud system allows the mobile operators to scale network resources up and down to meet QoS requirements. Meanwhile, this also improves device resource utilization. Second, the adoption of the SDN concept to control traffic through a series of middle boxes (i.e., SFC) offers higher flexibility in service delivery from the mobile network to the Internet, and vice versa. The traffic from each subscriber only traverses through a series of middle boxes, as defined by for that particular subscriber.

\section{SDVMN Taxonomy}

The first step in understanding the concept of SDN and virtualization in a mobile network is to elaborate a classification using a taxonomy that simplifies and eases the understanding of the related domains. In the following section, we define the taxonomy of SDN and virtualization in mobile networks according to the current solutions. Our proposed 
taxonomy provides a hierarchical view and classifies the current approaches, corresponding to each part of mobile network architecture: mobile radio access network, mobile backhaul network, mobile core network, IMS system and service function chaining in the mobile network. In the following, we elaborate our proposed taxonomy.

\subsection{Radio Access Network}

At this level, the main issues identified in the literature are radio resource management, radio resource sharing, traffic management, and some use cases that will illustrate the benefits of SDN and virtualization in the mobile network.

\subsubsection{Radio Resource Management (RRM)}

Radio resource management (RRM) involves strategies and algorithms for controlling transmit power, channel allocation, and data rate. The object is to utilize limited radiofrequency spectrum resources and base station hardware resources as efficiently as possible. Works tackling problems regarding RRM focus on two classes of solutions: resource virtualization and resource abstraction.

- Resource virtualization The RAN functions are separated and partially centralized into the cloud system. For example, the main functions of the base station can be divided into baseband and radio processing. The baseband functions of several base stations are combined and virtualized on the general purpose processors to perform baseband processing. In this way, this solution brings several benefits, such as low power consumption, efficient hardware resources, and throughput maximization. The research works in this direction have attempted to determine which functions should be centralized and virtualized on the cloud and deployment model, such as fully centralized or partially centralized.

- Resource abstraction The base stations in a geographical area are abstracted as a big base station or a big cell that consists of a RAN controller and radio elements. The SDN-RAN controller will dynamically schedule and allocate radio resources to each radio element. The works in this direction focus on methods of allocating radio resources from a radio resource pool in the controller.

\subsubsection{Radio Resource Sharing}

As surveyed in [39], many sharing solutions are being applied into mobile network. They can be active or passive sharing approaches. By sharing resources in a radio access network, mobile network operators (MNOs) can reduce their CAPEX and OPEX. All of these advantages can help an operator to better position its business within the overall competitive environment. When the mobile network is intended to be redesigned with SDN and virtualization, two new technologies can bring more solutions for radio resource sharing in radio access networks. As a consequence, MNOs can further increase their revenue. In order to support resource sharing among the MNOs at the radio access level, works have proposed two solutions:

- Slicing This class focuses on FlowVisor-based solutions to slice a radio access network infrastructure into virtual radio access networks for different mobile operators. For 
example, this solution deals with slicing 3D grid resources, including time, frequency, and radio elements.

- Virtual base station This class relies on Hypervisor-based solutions to create the virtual eNodeB, which uses the physical infrastructure and resources of another eNodeB, depending on requests from the MNO.

\subsubsection{Traffic Management}

Traffic management is an important issue for mobile networks. Managing traffic efficiently can improve throughput, alleviate network congestion, optimize resource use, and enhance quality of service. At the radio access layer, works tackling traffic management focus on two main mechanisms: data offloading and load balancing.

- Traffic offloading The works in this direction propose new data offloading mechanisms based on the SDN concept, such as programmable policy-based offloading and wireless network condition-aware offloading.

- Load balancing We found only one work that mentions using a centralized controller with knowledge of the entire network to balance a workload among base stations.

\subsubsection{Use Cases}

The works in this section focus on specific problems, new aspects, and mechanisms of the radio access network that can be solved and improved with the support of SDN and virtualization. These use cases cover machine-to-machine (M2M) and device-to-device (D2D).

\subsection{Mobile Backhaul Network}

At this level, the works mainly concentrate on solving resource sharing issues and introduce some use cases of SDN and virtualization in the backhaul network.

\subsubsection{Backhaul Network Resource Sharing}

In addition to cutting down CAPEX and OPEX, resource sharing at the backhaul level can help network operators to recover from network failure or link congestion. Works in this category are targeted at solutions based on SDN and slicing mechanisms to create multiple virtual backhaul networks and allow one operator to share an amount of its own resources with another operator. In other words, these solutions helps re-direct mobile traffic from the communication links of one network operator to another for the purpose of load sharing in heavy traffic or link failure conditions.

\subsubsection{Use Cases}

The works in this category show some use cases in which the backhaul network can take advantage of SDN and virtualization, such as mobility management, congestion control, and traffic-aware reconfiguration. 


\subsection{Mobile Core Network}

In this part of the taxonomy, we target issues that belong to the core network part.

\subsubsection{Use Traffic Routing}

Routing protocol is one of the important issues when we evolve our mobile network using SDN and virtualization. There are two directions for evolving a mobile core network: revolutionary and evolutionary. The revolutionary approach radically changes the current architecture of a mobile core network by using SDN-based switches, and mobile network functions are redesigned and implemented in the SDN controller. The work dealing with this approach focuses on two main routing solutions: tag-based routing and flow-based routing. The evolutionary approach applies the SDN concept into a part of the traditional core network architecture. This evolutionary approach analyzes the traditional mobile network functions, such as PGW, SGW, MME, etc., and decides which functions should be implemented to the controller, and which should be implemented in the traditional dedicated hardware. The research works in this approach keep using traditional routing protocols in the mobile core network, i.e., GTP-based routing.

- IP-based routing packet routing relies on the destination IP address.

- Tag-based routing packet routing can be done using a multi-dimensional tag, including policy, base station ID, and user equipment ID.

- Flow-based routing packet routing relies on selected fields of the IP packet header.

- GTP-based routing packet routing is based on GPRS Tunneling Protocol. However, these GTP tunnels are set up by the SDN controller in a central manner instead of systematic establishment as in the traditional mobile network.

\subsubsection{Core Network Resource Sharing}

Similarly to resource sharing problems in radio and backhaul networks, resource sharing at the core network is considered as a benefit of SDN and virtualization in the mobile network. Work in this direction focuses on slicing techniques to create multiple virtual core networks shared among multiple network operators. The work proposes architectures that extend from FlowVisor to slice cellular core network resources.

\subsubsection{Traffic Management}

Similarly to the radio access level, works dealing with traffic management at the core network cover load balancing, traffic offloading, and monitoring.

- Load balancing works in this category propose SDN-based solutions to move traffic load among SGWs or redirect data traffic from mobile networks to the Internet directly.

- Traffic offloading works in this category propose solutions that deploy SDN infrastructure in the mobile core network to offload selected traffic to cloud data centers.

- Monitoring works in this category propose solutions that integrate SDN-enabled monitoring platforms into the current mobile networks. These solutions allow configuring measurement devices dynamically and easily according to the requirements of operators. 


\subsubsection{Use Cases}

The research in this category covers several use cases of the mobile core network with the support of SDN and virtualization. These use cases are mobility management, content delivery network (CDN) or information centric network (ICN), MTC traffic (D2D, M2M), and security.

\subsection{External Networks}

At this level, the major issues focus on the interfaces between the mobile network and external packet data networks. The works in this category cover several use cases of SDN and virtualization in delivering applications and services between mobile network and the external networks.

\section{SDVMN: Current Approaches}

As defined in the taxonomy tree in Fig. 5, in this section, we present a survey on the most relevant research initiatives and current approaches adopting SDN and virtualization into today's mobile network.

\subsection{Radio Access Network}

\subsubsection{Radio Resource Management}

One of the most prominent objectives of efficient radio resource management is to eliminate inter-cell interference. Inter-cell interference management helps to avoid the overlapping between cells so that the user throughput is improved. The drawback of inter-cell interference management in existing mobile networks is that it is performed in a distributed fashion. Thus, the techniques such as inter-cell interference coordination (ICIC), enhanced ICIC (eICIC) or coordinated multi-point transmission/reception (COMP) are highly complex and suboptimal. However, with the SDN concept, this task is done easily by an SDN controller in a central manner. Indeed, with the global view of the whole network, RRM function on top of the SDN controller can construct an interference map between base stations and thus can mitigate the inter-cell interference easily. In addition, through the SDN controller, any RRM upgrades can be achieved independently from the base station hardware. In the following we show how the radio resource is managed and allocated in the RAN part of SDVMN.

5.1.1.1 Resource Virtualization Proposed by China Mobile [40] in 2011, the C-RAN concept spread widely in recent years as the hottest topic in the radio access part of mobile networks. Unlike the classical distributed base station system, in which a radio remote head $(\mathrm{RRH})$ entity and baseband unit (BBU) entity are tightly coupled, C-RAN represents a system of distant RRH entities connected using high bandwidth front-haul links to a single centralized BBUs pool on a cloud computing system. The radio signal from/to a particular RRH can be processed by a virtual base station, which is part of the processing capacity allocated from the physical BBU pool by the virtualization technology. On the other hand, the resource for an individual RRH is dynamically and flexibly allocated. For efficient 


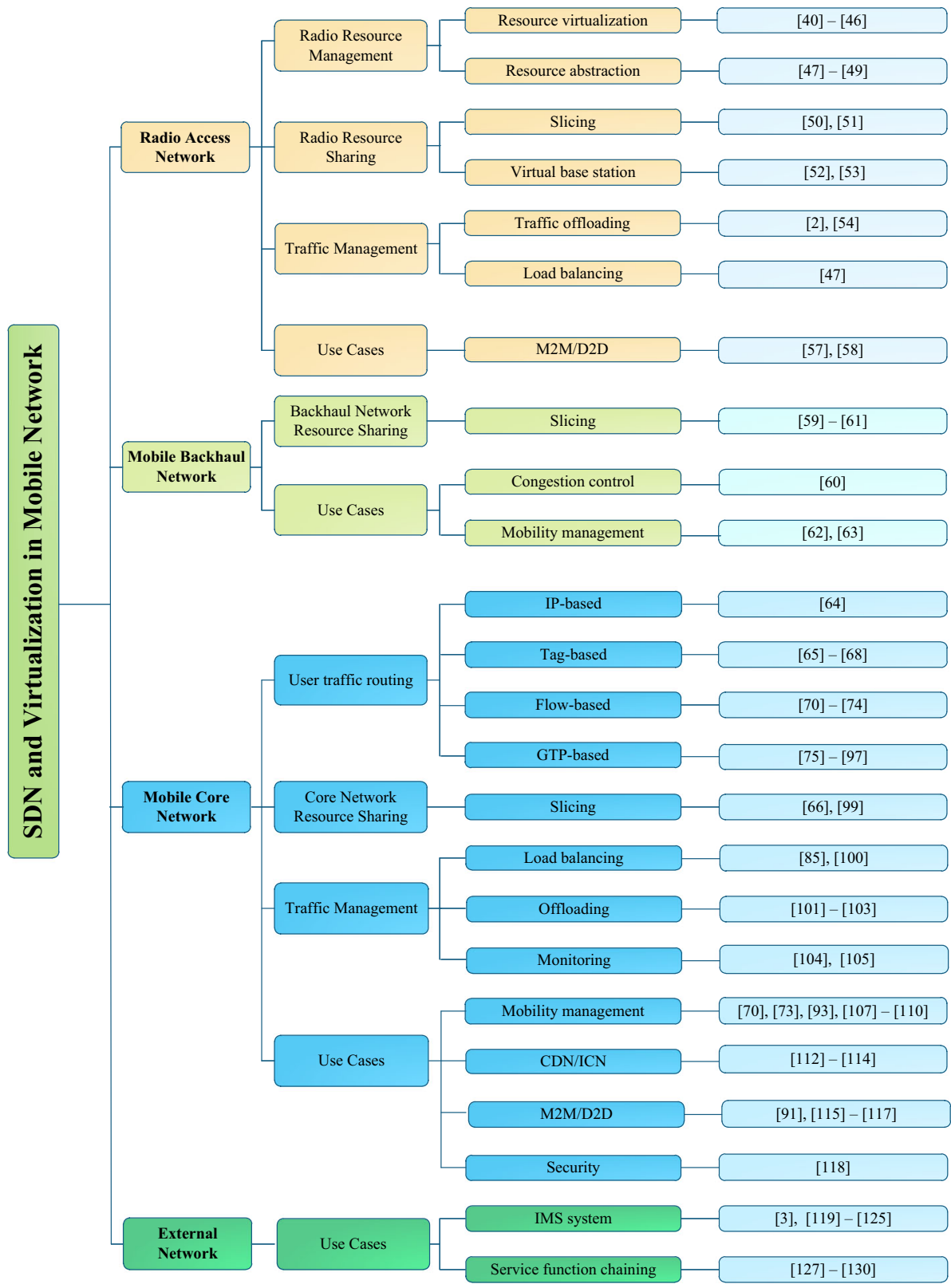

Fig. 5 Taxonomy and research works for future mobile networks based on SDN and virtualization

radio resource management in a multi-cells environment, C-RAN adopts the multi-cell joint radio resource management and cooperative multi-point transmission schemes. After the C-RAN concept was proposed, Hadzialic et al. [41] focused on known techniques to realize this concept. Checko et al. [42] made a survey that covers the state-of-the-art literature on C-RAN in the mobile network. This technology overview makes it easy for 
anyone who wants to understand the fundamentals of C-RAN and engage in research activities on C-RAN. The CONCERT [43] proposed a more flexible solution than the $\mathrm{C}-\mathrm{RAN}$ in which the baseband resources can be virtualized in a hierarchical manner with three levels: local, regional and central resource pools. In addition, the front-haul network of CONCERT architecture is SDN network.

Unlike C-RAN and CONCERT, the authors in [44, 45] proposed RANaaS, a general radio resource virtualization solution, when considering the trade-off between the full centralization and decentralization. It means RANaaS partially centralizes RAN's functionalities depending on the actual needs as well as network states.

Yang et al. [46] proposed OpenRAN, which is the integration of the C-RAN and SDN concepts. By doing so, the RAN becomes open, controllable, and flexible. OpenRAN contains three main parts: a wireless spectrum resource pool (WSRP), cloud computing resource pool (CCRP) and an SDN controller. The WSRP is composed of multiple physical remote radio units (pRRUs) to enable several virtual RRU (vRRU) to coexist in one shared pRRU via virtualization technology. The SDN controller plays a role of creating and dynamically optimizing vRRUs according to the requirements. It enables fair allocation and virtualization of radio spectrum, computing and storage resources to virtual access elements in heterogeneous RANs.

In summary, radio resource management with the concept of resource virtualization is mostly related to the migration of several RAN functions into a cloud computing system.

\subsubsection{Resource Abstraction Unlike the resource virtualization, SoftRAN [47], PRAN} [48] and V-Cell [49] introduce another new concept to tackle radio resource management, called resource abstraction. SoftRAN [47] proposed to rethink the radio access layer of current LTE infrastructure and focused on the control plane design by abstracting all the physical base stations (not only RRU) in a local geographical area into a virtual big base station. As a result, these physical base stations become simpler radio elements with minimal control logic and are controlled by a logically centralized controller. In SoftRAN, the radio resources are abstracted out as three-dimensional grids (3D grid) of time, frequency, and base station index or radio element index. Inside the controller of SoftRAN, there is a RAN information base (RIB) which is the core element of SoftRAN. The RIB consists of essential information to be updated by the controller including an interference map, flow records and network operator preferences. It is maintained by the controller and is accessed by the various control modules for radio resource management. PRAN [48] is an extension of SoftRAN which provides mechanism to dynamically reconfigure L1/L2 data plane.

While SoftRAN and PRAN focus on the abstraction of base stations and do not support multi access technology (Multi-RAT), V-Cell [49] targets cell abstraction with the ability to support multi-RAT and heterogeneous cells (macro, Pico, and femto). These cells are abstracted as a single big macro cell, and the radio resources for them are considered as a single pool of resources that is managed by a logically centralized software defined network controller (SD-RAN controller). The radio resources in V-Cell are abstracted out in a resource pool through a 3-dimensional matrix including time, frequency and space. The main role of the SD-RAN controller is to allocate radio resources, management limited spectrum, interference and power allocation and balance load across the entire pool of physical cells inside the V-Cell. In summary, the object of two approaches is to abstract the radio resources and manage them by using an SDN controller in a central manner. 


\subsubsection{Radio Resource Sharing}

5.1.2.1 Slicing In order to share radio resources among different mobile operators, one slicing technique was introduced in [50]. RadioVisor [50] extends the FlowVisor concept to allow each controller to access a slice of the radio access infrastructure, in particular SoftRAN architecture [47]. RadioVisor enables traffic to be steered to and from the controllers of virtual operators. It isolates radio resources, control channel messages, and CPU resources among various virtual operators. RadioVisor architecture is composed of a device and application to slice mapping, the radio resource allocation and isolation function, and slice manager. The first component is the traffic to slice mapping at RadioVisor and radio elements. The second component performs a heuristic algorithm to allocate the resource to slices in a fairness manner according to resource requests and the service agreements. The last component is in charge of slice configuration, creation, modification, deletion and multi-slice operations. It interacts with each slice through an API provided by RadioVisor. Each slice is defined based on operator, device, subscriber, and application attributes.

While RadioVisor presented a general concept for creating radio access network slices for different mobile operators, Spapis et al. [51] introduced a complete functional architecture to only share the spectrum resources among mobile network operators. Depending on the needs as identified by their operations, administration, maintenance (OAM) modules, the spectrum resources will be allocated from a central spectrum pool to eNBs in the RAN through RRM commands. In addition, the authors also presented a potential implementation model based on the combination of SoftRAN architecture [47] and MobileFlow architecture [75].

5.1.2.2 Virtual Base Station Another approach for resource sharing was proposed in [52, 53], which create virtual base stations from the shared physical base stations of multiple network operators. Zaki et al. [53] proposed a sharing solution based on the virtualization of eNodeB in the LTE access network. A key component is called Hypervisor, which is located on top of the physical eNodeB resources and is in charge of allocating these resources to each virtual eNodeB (VeNB) according to the request of different MVNOs. Costanzo et al. [52] presented OpeNB, which is also based on the virtualization of eNodeB in the LTE access network. However, the OpeNB architecture is more flexible by leveraging SDN and Openflow technologies. In OpeNB, the virtualization of eNodeB can be handled dynamically based on network state and some predefined agreements among various operators. It enables an operator to hire on-demand the physical infrastructure and resources owned by other operators. The OpeNB architecture consists of two types of physical elements: NAeNBs, OpeNBs, and two types of software entities: a main controller (MC) and OpeNB controller (OC). NAeNBs are NV aware eNodeBs, which can forward the NV signaling from the MC toward an OpeNB. OpeNBs are eNodeBs that are controlled by the MC and have the ability to create VeNBs. OpeNB sharing between multiple operators is done by NV procedure triggered from the MC. The OC located inside an OpeNB is responsible for triggering VeNBs upon the request from the MC. These works are summarized in Table 1. 
Table 1 Survey on resource sharing solution in SDVMN

\begin{tabular}{|c|c|c|c|}
\hline Reference & $\begin{array}{l}\text { Network } \\
\text { part }\end{array}$ & $\begin{array}{l}\text { Solution } \\
\text { type }\end{array}$ & Solution description \\
\hline $\begin{array}{l}\text { Radiovisor } \\
\text { [50] }\end{array}$ & RAN & Slicing & $\begin{array}{l}\text { Create slices of radio access infrastructure. Each slice is } \\
\text { defined using predicates on operator, device, } \\
\text { subscriber, and application attributes }\end{array}$ \\
\hline $\begin{array}{l}\text { Spapis et al. } \\
\text { [51] }\end{array}$ & RAN & Slicing & $\begin{array}{l}\text { a complete functional architecture to only share the } \\
\text { spectrum resources among mobile network operators. } \\
\text { Depending on the needs as identified by their OAM } \\
\text { modules, the spectrum resources will be allocated } \\
\text { from a central spectrum pool to eNBs in the RAN } \\
\text { through RRM commands }\end{array}$ \\
\hline $\begin{array}{l}\text { Costanzo et al. } \\
\text { [52] }\end{array}$ & RAN & $\begin{array}{l}\text { Virtual base } \\
\text { station }\end{array}$ & $\begin{array}{l}\text { Virtual eNodeBs (VeNBs) are created dynamically } \\
\text { from shared physical eNodeBs' resources with the } \\
\text { assist of SDN controller }\end{array}$ \\
\hline Zaki et al. [53] & RAN & $\begin{array}{l}\text { Virtual base } \\
\text { station }\end{array}$ & $\begin{array}{l}\text { VeNBs are created from shared physical eNodeBs' } \\
\text { resources according to the request of different mobile } \\
\text { virtual network operators }\end{array}$ \\
\hline $\begin{array}{l}\text { Philip et al. } \\
\text { [59-61] }\end{array}$ & $\begin{array}{l}\text { RAN and } \\
\text { Backhaul }\end{array}$ & Slicing & $\begin{array}{l}\text { Backhaul infrastructure in which access and } \\
\text { aggregation backhaul nodes are incorporated with } \\
\text { openflow protocol is shared by using a slicing } \\
\text { mechanism }\end{array}$ \\
\hline CellVisor [66] & Mobile core & Slicing & $\begin{array}{l}\text { Using slicing technique to share mobile core network } \\
\text { infrastructure in the revolutionary mobile architecture }\end{array}$ \\
\hline $\begin{array}{l}\text { MobileVisor } \\
\text { [98] }\end{array}$ & Mobile core & Slicing & $\begin{array}{l}\text { Using slicing technique to share mobile core network } \\
\text { infrastructure in the evolutionary mobile architecture }\end{array}$ \\
\hline
\end{tabular}

\subsubsection{Traffic Management}

5.1.3.1 Traffic Offloading Mobile traffic offloading refers to the movement of data traffic from a mobile network to a Wi-Fi network. In a mobile network, the traffic offloading is done by an access network discovery and selection function (ANDSF). This entity is used for discovering wireless networks close to the mobile user and then performing the Wi-Fi offload. In [2], the author said that the mobile traffic offloading can be done by SDN since the SDN controller can dynamically control the traffic in a mobile network based on various trigger criteria including individual flow rate, aggregate flow rate, application type, available bandwidth, etc. In [54], the authors introduced a new architecture for offloading data traffic from a mobile cellular network to a Wi-Fi network by converting network resource management (NRM) and RRM functions to software modules running on an SDN controller. The RRM application is responsible for collecting radio access network conditions such as traffic load or cell capacity while NRM (integrated PCRF) stores the subscriber and application information. In this architecture, the SDN controller is in charge of composing information of these two modules and creates a single set of policies and rules which are then installed to the local control agents in the network gateway and the RAN. This architecture enables the SDN controller to gather the real-time network condition and then decide suitable offloading policies.

5.1.3.2 Load Balancing Consider the case where one base station is overloaded with many clients while a nearby base station is free of serving any client. Due to the nature of 
the distributed control plane in the traditional RAN network, there is no mechanism to balance the traffic load between two such base stations. In [47], the authors proved that the load balancing can be facilitated by using the SDN controller, which has the global view of the entire network and workload. Indeed, the SDN controller will trigger the handover of edge users to balance the load between one base station and its neighbors.

\subsubsection{Use Cases}

Since SDN and virtualization brings a number of benefits in a radio access network, many works have proposed to adopt them into different application domains including dealing with the machine to machine (M2M) and device to device (D2D) traffic. In the following, we will review these use cases.

5.1.4.1 Dealing with M2M and D2D Traffic M2M [55] and D2D [56] are two new communication types in mobile networks. The M2M and D2D over mobile cellular network refer to the communication between two mobile nodes without traversing to the base station or mobile core network. Instead, these devices often communicate with each other through a M2M or D2D gateway. The authors in [57] proposed a framework that combines the SDN-based cellular mobile network with M2 M. The main purpose of this paper is to flexibly reconfigure the cellular network based on the monitoring information received from M2M devices. The M2M sensing devices monitor the surrounding area of eNodeBs and send the sensing data to the M2M server via the IP backbone network. The M2M server will alert the SDN control software to reconfigure the network assessments and resource allocation to avoid a disaster in the network. In order to control the congestion exposed by the M2M communication in the LTE network, the authors in [58] proposed congestion control methods based on SDN and Openflow. To verify this method, the authors deployed a test-bed framework using NetFPGA and Openflow platform.

\subsection{Mobile Backhaul Network}

\subsubsection{Backhaul Network Resource Sharing}

As discussed above, the reduction of CAPEX/OPEX and the improvement of network efficiency and utilization are two objectives of resource sharing in mobile networks. At this part, the authors in [59-61] proposed a solution for backhaul infrastructure sharing by using a slicing mechanism and incorporating Openflow protocol within the access and aggregation nodes. The slicing mechanism is done by extending the FlowVisor concept. As a result, an operator can lease the backhaul network of another operator in cases of link congestion and heavy traffic condition.

\subsubsection{Use Cases}

In the following, we describe briefly some use cases in mobile backhaul with SDN and virtualization.

5.2.2.1 Congestion Control Philip [60] proposed Openflow-based backhaul network architecture which allows one operator to share its backhaul resources with others. This solution provides mobile operators with backup links in cases of link congestion or heavy 
traffic condition. Thus, mobile operators can achieve better resource utilization within their own backhaul network.

5.2.2.2 Mobility Management In order to improve the mobility management efficiency in cases of inter-eNodeB handovers, the authors in [62] proposed SDMA, a semi-distributed mobility anchoring in LTE backhaul access network based on Openflow and SDN technologies. In this architecture, all backhaul access nodes are realized as Openflow switches and controlled by Openflow Controllers via Openflow protocol. Furthermore, the concept of service VLAN (S-VLAN) id and customer VLAN (C-VLAN) id is used to separate the traffic of the users under different Openflow controllers. Then a detail of handover procedure including initial attach, handover under the same controller or different controllers was defined. Unlike [62], the authors in [63] tried to fully realize the concept of SDN into the entire mobile backhaul network. They introduced a distributed SDN control plane. The management plane, including MME, HSS, PCRF, and billing system, is implemented within VMs that can communicate with SDN controllers. As a case study of mobility management, the authors indicated that the new architecture reduces not only the power consumption of the UE but also the signaling message overhead between entities at the backhaul side.

\subsection{Mobile Core Network}

\subsubsection{User Traffic Routing}

As defined in the taxonomy tree above, there are three kinds of mechanisms for routing the user traffic in SDVMN: tag-based, flow-based or IP-based, and GTP-based. For each method, we show how the current mobile architecture is redesigned with them as depicted in Fig. 6.

5.3.1.1 IP-Based Routing The authors in [64] considered IP-based routing in virtualization-based LTE EPC architecture (vEPC). In this architecture, the control plane and user plane of EPC entities also are decoupled from each other. Two new entities, namely the EPC edge router (EPC-E) and core router (RTR), were introduced. EPC-E is a router which locates at the same place as the SGW and terminates GTP tunnel between RAN and the core part. It maintains routing information of every UE that is notified by the control plane. RTRs are regular IP routers that are configured from the vEPC's control plane by a routing protocol like BGP. In other words, BGP is used as protocol for updating the routing in formation in EPC-E and RTR from vEPC's control plane as well as for routing between EPC-E and RTR in the data or user plane.

5.3.1.2 Tag-Based Routing Li et al. [65] proposed SoftCell, which aims at designing a revolutionary architecture for mobile networks to support numerous fine-grained policies in a scalable manner. The SoftCell architecture is composed of commodity switches, which are simpler and cheaper than specialized network elements (SGW, PGW) and just perform packet forwarding, and relegate complicated packet processing to middleboxes; a set of middleboxes, which act as transcoders, web caches or firewall; access switches, which classify fine-grained packets on traffic from UEs; and a central controller, which is responsible for computing and installing switch-level rules based on subscriber attributes and application types. In order to deal with the challenges of data explosion in the data 


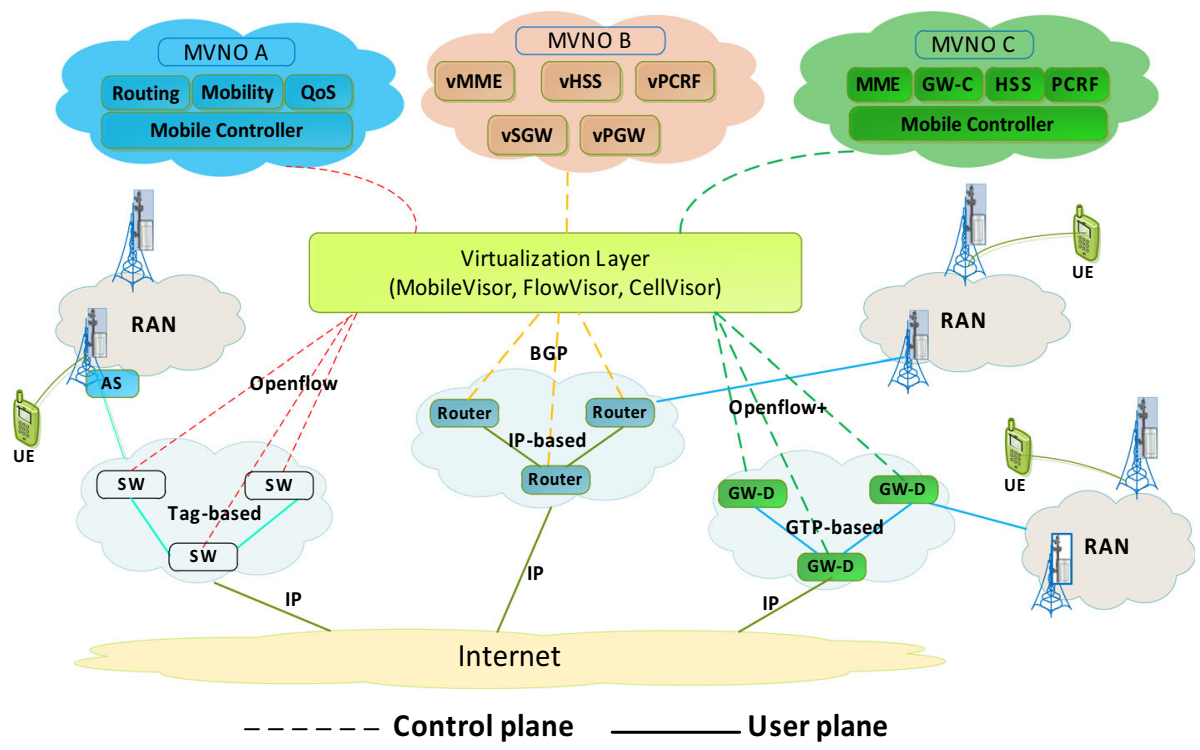

Fig. 6 Overview of SDN and virtualization solution in a mobile packet core network

plane or the scalability problem in the flow table, the authors proposed the use of multidimensional aggregation and tag-based routing. In particular, the forwarding rules in SoftCell are aggregated on three dimensions: policy, location ID, and UE ID. For example, the policy tag allows for aggregating flows that share a common policy. CellSDN [66], the prior work of SoftCell, leverages SDN into both access and core networks. In this work, the author mainly focused on describing use cases that can benefit from CellSDN, such as network control and billing monitoring, seamless subscriber mobility, and remote control of the base station. The routing mechanism is the same as SoftCell by using tag-based routing. Moradi et al. $[67,68]$ proposed SoftMoW to address the challenges in a very large cellular network or mobile WAN. The challenges derive from suboptimal routing, lack of support for seamless inter-region mobility, scalability, reliability, and the rise of new applications (e.g., M2M, IoT). SoftMoW is composed of distributed programmable switches and middleboxes instead of expensive and inflexible gateways (SGW, PGW). SoftMoW is constructed in a hierarchical control plane for the core and radio access networks with $\mathrm{L}$ levels. The physical data plane is divided into several logical regions, each of which is controlled by a leaf controller. Leaf controllers abstract their topologies and expose logical components to the next level, including a gigantic switch (G-switch), an abstraction of all physical switches; a gigantic base station (G-BS), an abstraction of a group of physical base stations; and a gigantic middlebox (G-Middlebox). As a consequence, the entire network is controlled by a highest-level parent controller at the root region. The routing mechanism in SoftMoW is also performed by an alternative method of tag-based routing called label-based routing. The parent controller pushes a global label into each traffic group while the child controllers perform label swapping at ingress and egress switches. Openflow protocol is assumed as the southbound interfaces in CellSDN and SoftCell as well as SoftMoW architecture. 
5.3.1.3 Flow-Based Routing In [70, 71], the authors proposed CROWD, an SDN-based solution for dense heterogeneous wireless networks. The main target of this solution is to deal with various challenges in dense heterogeneous wireless network in terms of mobility management, interference management, and energy consumption. The CROWD architecture consists of two-tier SDN controller hierarchy with two types of controllers: a local controller (CLC) and regional controller (CRC). The CLC requires data from the network at a more granular time scale while the CRC requires aggregate data from the network and takes responsibility for management of the CLCs. The data plane of CROWD architecture is composed of DMM gateways (DMM-GWs), which are interconnected using an Openflow-based transport network. Therefore, the data traffic will be routed using a flow-based routing mechanism.

Trivisonno et al. [72, 73] proposed an SDN-based plastic architecture for 5G networks consisting of unified control plane and a clean-slate forwarding plane. The control plane is represented through three logical controllers: edge controller I for processing signaling messages for access nodes and installing forwarding rules into non-access nodes, edge controller II for processing signaling messages between UEs and access nodes, and an orchestration controller which is responsible for resource management and allocation of both $5 \mathrm{G}$ control and data plane entities. The data traffic between access and non-access nodes are routed using a flow-based routing mechanism. In addition, the authors also showed the backward compatibility with the legacy systems.

The authors in [74] also proposed an approach for LTE mobile core network relying on the concept of SDN. In this architecture, the MME entity still exists and is responsible for mobility management. The MME communicates with an Openflow controller to trigger forwarding path setup in Openflow switches, routers according to the UE's state. The routing in the data plane is relied on flow-based routing mechanism.

5.3.1.4 GTP-based Routing Compared to the above approaches, the GTP-based routing approach is the most popular solution for routing user data packets in SDN and virtualization-based mobile core networks. This approach refers to the evolution of mobile core networks where the tunnel between core network entities is still kept and the tunnel setup is facilitated by the use of an SDN controller. MobileFlow [75] proposed a software-defined mobile network (SDMN) architecture. In MobileFlow, the control and data plane are also decoupled from each other. Two main components of MobileFlow are the MobileFlow forwarding engine (MFFE) and MobileFlow controller (MFC). MFFEs are interconnected by an underlying Openflow-based transportation network. MFFEs must support carriergrade functionality, such as tunnel processing and charging. The MFC has some necessary functions for managing the entire network, such as topology discovery, network resource monitoring or network resource virtualization. In addition, the MFC consists of function blocks for tunnel processing, mobility anchoring, routing and charging. The mobile network applications, including the control functions of all current EPC entities (e.g., eNB-C, SGW-C, PGW-C, MME, etc.) are implemented on top of the MFC. The MFC computes and sets up GTP tunnels into MFFEs by using a lightweight protocol called Smf.

Several proposals [76-80] leverage the SDN concept into mobile core gateways. In [76], the authors made an analysis of all mobile core gateways' functions and then mapped them into four alternative deployment frameworks based on SDN and Openflow including full cloud migration, control-plane cloud migration, signaling control cloud migration and scenario-based cloud migration. The authors clearly presented the pros and cons of each deployment decision. In addition, several SDN frameworks for GTP tunnel processing and 
charging control function are also proposed. Next, in [77] these authors continue their work in [76] by addressing the function placement problem. In this work, the authors grouped four deployment models in [76] into two main categories: a virtualized gateway (NFV) and decomposed gateway (SDN). The first category refers to fully virtualizing SGW and PGW into a data center, and an off-the-shelf network element (NE) is used to direct the data traffic from the transport network to the data center. The second category refers to decomposing gateway functions, meaning that only the control plane function is shifted to the data center and integrated with SDN controllers while the data plane is processed by enhanced SDN network elements (NE+). To find the most optimal deployment solution, the authors formed a model by taking the control-plane load and data-plane latency into account, and then tried to minimize these parameters. By doing so, the operators will have a tool to make their own deployment decision: virtualizing all gateways or decomposing all gateways or a combination of two. As an extension of the work in [77, 78] proposed power saving models to maximize power savings by adapting the datacenter operation according to the time-varying traffic patterns and datacenter resources. The authors in $[79,80]$ similarly adopted SDN and NFV into EPC S/PGWs. In these works, the control function of an $\mathrm{S} / \mathrm{P}$ integrated gateway $(\mathrm{S} / \mathrm{PGW})$ is decoupled from the user plane. While virtualized $\mathrm{S} / \mathrm{PGW}$ control is realized as VMs in a cloud computing system, S/PGW user plane can be realized either by VM or dedicated hardware. The dedicated hardware is located possibly close to the access network and is responsible to fast path processing. Another network function implemented in VM in cloud is router functionality. It is used to run routing protocol and advertise UE IP prefixes via the SGi interface. The last element is the SDN controller, which is responsible for allocating UE IP addresses and GTP TEIDs and installing UE specific flow entries to the switch during an attachment procedure and modifies them during a handover. The main objective of this work is to dynamically switch the GTP termination point of an active session between the cloud and fast path in dedicated devices.

Regarding the implementation aspect, several efforts have been made to design appropriate protocols for software defined mobile networks [81-84]. Considered the first approach that adopted the SDN and Openflow concept into the LTE EPC environment, the authors in [81-83] introduced an evolution of mobile core network architecture based SDN and Openflow, which allows the entire control plane to be moved into a data center. In these works, the authors mainly focused on enhancements of Openflow 1.2 for supporting the process of GTP tunnel in the cloud-based mobile core network. While [81, 82] clearly describe how to manipulate Openflow 1.2 to implement GTP TEID routing in EPC architecture, [83] takes 3G packet core network architecture into account. Alternatively, [84] presented the detail design, implementation, and evaluation of a software defined telecommunication controller (OFC), switch (OFS) and protocol (OFP) according to the latest Openflow standards [6] (i.e., Openflow version 1.4) in regard to the EPC. The software defined telecommunication network (SDTN) architecture presented in this work also relies on the separation of the control plane and user plane. The design of OFS in SDTN is done by using a table-lookup pipeline with multiple table support, virtual port for GTP/GRE matching and Openflow agent for communicating with the OFC. The control functions, such as PGW-C and SGW-C, can be deployed as higher layer network applications upon the controller.

Other approaches [85-88] leverage Openflow into the LTE/EPC architecture with some procedure analyses. In [85, 86], the authors introduced a new control plane in 3GPP LTE/ EPC architecture for supporting on-demand connectivity services such as load balancing and resiliency and then proved the reduction of signaling load by providing a procedure 
analysis. The architecture in these works only adopted SDN and Openflow into SGW entities. It means that only the control function in SGW is separate from the data forwarding function in SGW. The SGW control function (SGW-C) and MME function are packaged as applications running on top of the Openflow controller. Similarly, the authors in $[87,88]$ also made a procedure analysis in Openflow-enabled LTE/EPC architecture and evaluated in terms of signaling load. However, compared to [86, 88] proposed a solution that is fully realized in SDN and Openflow technologies. In other words, in [88], all control functions of both SGW and PGW are packaged together with MME as applications on top of the mobile controller. By doing so, the signaling load can be reduced more than that in [86]. These approaches still require the GTP processing at the data plane elements.

Instead of redesigning the mobile core network, K. Gomez et al. [89, 90] present a new distributed entity, named a flexible management entity (FME), which leverages virtualized EPC functionalities in 4G LTE architecture. The main goals of FME are to embed the most fundamental EPC operations close to the radio access network (eNB) and make coexistence possible between virtual and physical EPCs. The FME is composed of a virtual EPC, link management unit (LMU), routing management unit (RMU) and topology management unit (TMU). The virtual EPC represents all normal EPC operations: user plane processing by EPC-Agent (EPC-A) and control plane processing by MME-Agent (MME-A). In summary, the FME units (LMU, RMU, and TMU) interact in order to create and maintain the vS1 interface while FME agents (EPC-A and MME-A) create and maintain the bearers. ProCel [91] is another approach that does not modify the current EPC architecture. Instead, ProCel introduces new entities, including ProCel switches and a ProCel controller, to dynamically process the traffic whether it goes through the cellular core network or can be steered down to a fixed IP network. In the control plane, the ProCel controller computes and installs forwarding rules in ProCel switches based on policies from operator databases. In the data plane, ProCel switches perform packet classification with three categories: core flow, to be routed toward mobile core networks; non-core flow, to be steered down to fixed networks; and controller-traffic, to be forwarded to the ProCel controller. Some proposals just introduced SDN and Openflow in the transportation network [92, 93] while other parts are still kept the same as the current mobile network architecture. The routing mechanism in the EPC is still based on the GTP tunnel.

Regarding the virtualization aspect only, works in [94-96] discuss the potential benefits of NV, NFV and cloud computing concepts in the mobile core network. The authors in [94] presented the vision of the Mobile Cloud Network project [14], an EU FP7 Large-Scale Integrating Project funded by the European Commission. This work showed a new architecture relying on the cloud computing concept for the entire mobile network. In the mobile core part, several virtualization strategies and models are proposed. Hawilo et al. [95] presented the challenges and implementation of NFV in next generation mobile networks. In this paper, the authors proposed an approach to group several vEPC entities based on their interactions, workload and functionalities to achieve less control-signaling traffic and less congestion in the data plane. There are four grouping strategies, namely segment one, segment two, segment three, and segment four. Segment one is the group of MME and HSS front-end (HSS FE), which implements all HSS function without containing a user information database. Segment two is the group of SGSN and HLR FE. Segment three is the group of PGW and SGW. And the segment four is the group of UDR, PCRF, online charging system (OCS and offline charging system (OFCS). SoftEPC [96] introduced a general purpose node (GPN), which leverages the cloud technology concept into the EPC architecture. A GPN is a core-class server which has a hypervisor to provide virtual instances of EPC entities. In this work, the authors' object is to analyze the 
performance of utilizing the softEPC and to illustrate the improvement of network utilization and service delivery enhancement provided by softEPC. Since the mobile network functions, such as SGW, PGW and MME, are kept unchanged in the cloud computing system compared to the traditional mobile network, the GTP tunnels are still used for packet routing. The authors in [97] proposed a new telecom architecture by extending the concept of SDN. In this architecture, the control plane consists of MME and the control functions of SGW and PGW while the user plane is composed of SDN forwarding entities (SDN-FEs). The authors used the term "vertical forwarding" to refer to the use of GTP tunneling over an SDN forwarding domain. It means that SDN-FEs in their architecture are enable to encapsulate and decapsulate GTP header of the packet. Table 2 summaries and compares these aforementioned works on user traffic routing in SDVMN.

\subsubsection{Resource Sharing}

Li et al. [66] proposed a slicing technique, called CellVisor, in the revolutionary SDNbased core network to create multiple virtual core networks. Giang et al. [98] considered a general solution, called MobileVisor, in both evolutionary and revolutionary SDN-based core networks. MobileVisor allows multiple virtual operators with various platforms to share the same physical core network infrastructure.

\subsubsection{Traffic Management}

Load Balancing Siwar et al. [85] proposed an SDN-based architecture that implements MME and SGW control functions in the controller. This architecture can move a workload from an overloaded SGW to another. Ghazisaeedi et al. [99] proposed an Openflow switching system, which includes Openflow switches connected to SGW and directly to the Internet. These Openflow switches can detect HTTP traffic and forward it directly to the Internet. This reduces the burden for the mobile core network.

Traffic Offloading SMORE [100], MOCA [101], and SOFTOFFLOAD [102] are SDNbased offloading mechanisms in the mobile network. Both MOCA [100] and SMORE [101] proposed to redirect selected traffic to a cloud platform. MOCA suggested some modifications to MME and cloud-based SGW while SMORE can handle offloading using SMORE-SDN infrastructure without requiring any modifications to the entities in the mobile network. SOFTOFFLOAD [102] used an SDN-based network running SoftOffload protocol to offload traffic from cellular to Wi-Fi.

Monitoring Donatini et al. [103] developed an SDN-based measurement platform, which is integrated into the mobile network. This platform allows easy configuration of measurement devices and monitoring functions following the requirements of the operators. In [104], the authors proposed a monitoring architecture, which uses SDN-based network to connect monitoring ports and monitoring tool farm. This architecture allows adding more monitoring ports and tools if necessary.

\subsubsection{Use Cases}

5.3.4.1 Mobility Management The current mobility management in the mobile network relies on two central anchor points-SGW and PGW. It results in non-optimal routing path, high handover latency, and a single point of failure. Nowadays, distributed mobility management (DMM) [105] is considered as a promising solution to solve these above 


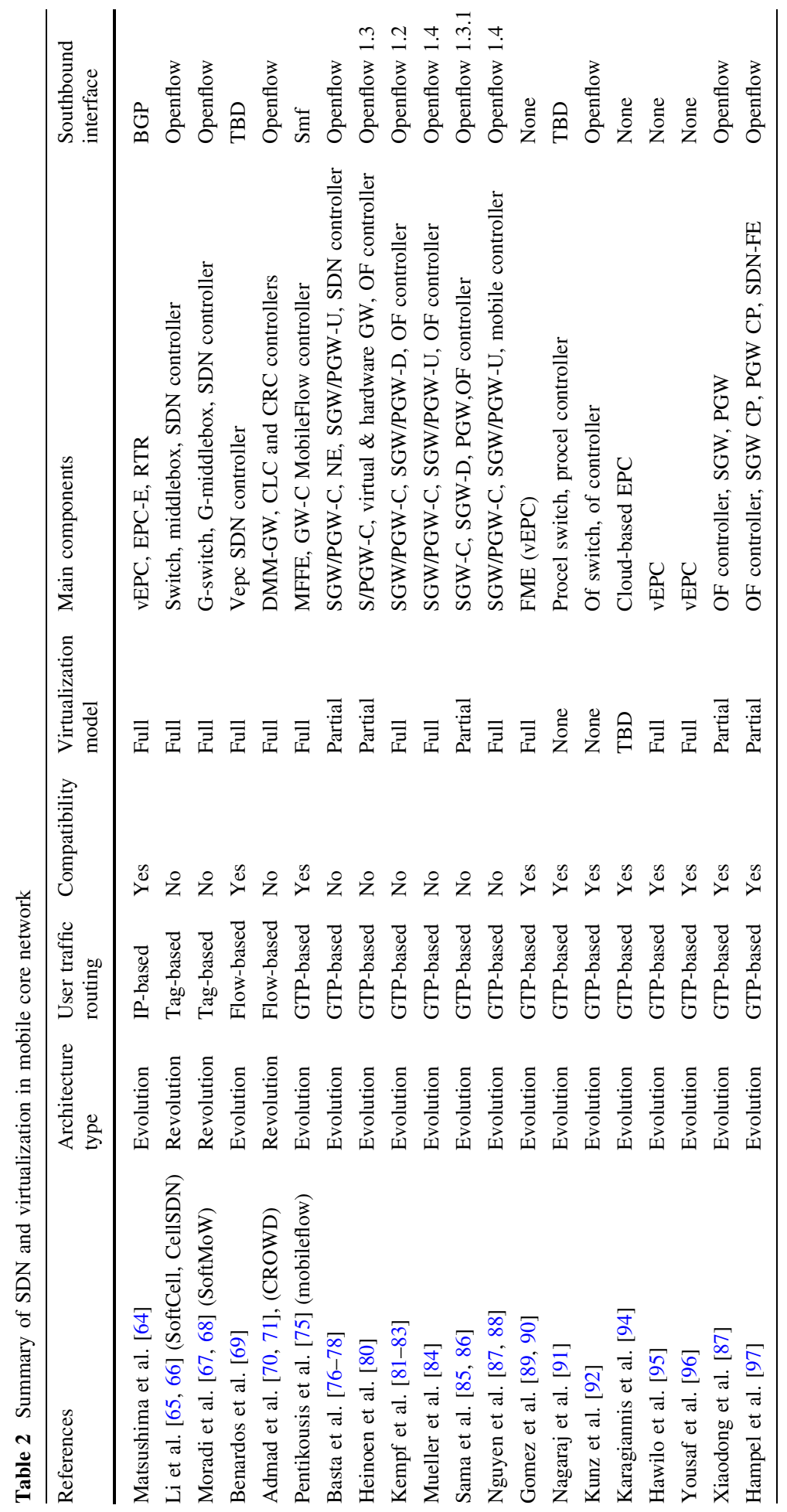


challenges. Inspired by the benefits of DMM, several proposals [70, 93, 106-108] tried to redesign mobile network architecture by leveraging SDN and Openflow with the support of DMM. The authors in [70] presented a DMM implementation in the CROWD architecture [71] which was discussed before, to optimize mobility management not only in WLAN networks but also in LTE networks. In this architecture, DMM-GW is the substitution of SGW and PGW and acts as the terminal's session anchor point. The mobility management logic functions are executed by control applications, either local or remote to the CLC. For example, in the intra-handover case, the CLC is responsible for computing the required match rules and data path modifications to forward the user packet to the selected DMMGW via the Openflow protocol. In the inter-handover, the mobility management task relies on both the CLC and the CRC. It is required to define new APIs to control the DMM-GWs at IP layer. The detailed design of this solution is described in [106]. Karimzadeh et al. [93, $107,108]$ presented a solution to support DMM in the virtual LTE system. The virtual LTE system is composed of virtual S/PGWs running on VMs that are hosted on data centers. A new DMM transportation network was introduced in these works. The SDN/Openflow controller is responsible for controlling both the DMM transport network and EPC transport network. The use of DMM in the virtualized LTE system can enable the session continuity for UEs and redirect the traffic in case the virtual S/PGWs are migrated from one data center to another. The detail performance evaluation is shown in [108].

Kuklinski et al. [109] presented several scenarios for handover management in an SDNbased mobile network. These scenarios include full centralized, semi-centralized, and hierarchical approaches. The third scenario is similar to the mobility management in CROWD approach [70].

Trivisonno et al. [73] which was discussed earlier also provides some procedures for handover management in their SDN-based 5G network architecture.

5.3.4.2 CDN/ICN The content delivery network (CDN) is a system relies on the traditional host-to-host communication model to deliver requested data to users based on their geographic locations with high content availability and performance. Information centric networking (ICN) [110] is the new technology for the future Internet, which advocates a redesign of the current Internet infrastructure around the concept of content rather than around the concept of addresses, as in traditional Internet infrastructure. Several proposals [111-113] investigated the cases of CDN and ICN in the SDVMN. Karimzadeh et al. [111, 133] proposed a solution, which integrates ICN into the virtualized LTE system to support service and VM migration. In this solution, all of the virtualized LTE entities are ICN capable, while the routers in the transportation network are non ICN capable. In [112], the authors focused on video delivery services in the SDVMN. The main purpose of this work is to integrate SDN with the LTE network element for optimal video streaming. Concretely, the authors used SDN switches in a transportation network and integrated an SDN controller with an MME element in the LTE network. Video-on-demand is taken as a use case to demonstrate the benefits of this architecture. Haw et al. [113] introduced a framework for efficient content delivery in an SDN-based LTE network. The authors integrated an SDN controller with MME and deployed SDN-based SGW/PGW and SDN switches in the data forwarding plane. A CCN gateway is used to distinguish the arrived packets whether forwarded to the Internet or CCN network.

5.3.4.3 M2M/D2D As discussed before, nowadays the MTC traffic including M2M or D2D is imposing challenges for current LTE mobile network. Several proposals [91, 114- 
116] have been proposed to support these types of communication over SDN and virtualization based LTE architecture as well as to solve the challenges. Samdanis et al. [114] proposed a solution to eliminate the signaling overhead by adopting the NFV concept to machine type communication (MTC) devices (i.e., D2D or M2M devices). This paper introduced the concept of virtual bear, which can be shared by a specified set of MTC devices and the virtualization of gateway devices. Yazici et al. [115] proposed a new programmable 5G control plane architecture which supports D2D communication between two UEs. In particular, it solves mobility management of UEs in case of the movement of individual UE or both. Tableb et al. [116] proposed LightEPC, a lightweight EPC to support the communication between two MTC devices. It is enable to dynamically create scalable instances of MTC functions on demand. Similar to [116], the research work [91], which was discussed previously, supports D2D communication via a D2D agent included in FME entity.

5.3.4.4 Security Liyangage et al. [117] investigated the security problem in the control channel of SDMN and then proposed a solution to prevent the control channel of the SDMN from some potential attacks (e.g., TCP SYN Dos attack, TCP reset attack). The SDMN architecture consists of an Openflow-based data plane and a network controller. The network controller includes all control functions such as MME, PCRF, HSS, AAA, etc. To deal with the aforementioned potential attacks, the authors introduced three new entities: a security gateway (SecGW), security entity (SEC Entity), and local security agent (LSA). SecGWs are located between the controller and the rest of the network and are responsible for IPsec tunnels establishment with Openflow switches and for authentication and registration of new switches. The SEC Entity is a new control entity located in the network controller and in charge of controlling SecGWs. The LSA is a security module that is implemented on the switch. It is mainly responsible for establishing the secure channel with SecGWs (Table 3).

\subsection{External Network}

\subsubsection{Use Cases}

External networks, such as the IMS system and the Internet, are used to provide services and applications to mobile users. In this session, we briefly review several approaches that adopt SDN and virtualization concepts to improve the service and application delivery.

5.4.1.1 IMS System In [3], the basic model for virtualizing the IMS system based on the NFV concept was introduced. The network functions of the IMS system are deployed in VMs, which have scalable hardware resources and can be dynamically relocated in cases of a VM's overload or failure. Thus, the operator desired service continuity and service availability can be obtained. Similarly, Lu et al. [118] also proposed a cloud-based IMS architecture, with a load balancing algorithm and a mechanism for dynamic resource allocation. Ito et al. [119, 120] proposed a new EPC/IMS system based NFV concept where each service can be processed by a particular virtual network to reduce the signaling load.

Several proposals [121-123] follow another approach, which integrates the Openflow into the IMS system. Ito et al. [121] used Openflow-based routing to migrate call session state in the IMS system, which allows minimizing the number of servers as well as restoring call sessions subject to the halted server. The authors in [122] designed a novel QoE-aware IMS-based IPTV network architecture, deployed with the underlying Openflow 
Table 3 Summary of use cases in SDVMN

\begin{tabular}{|c|c|c|c|}
\hline References & $\begin{array}{l}\text { Use case } \\
\text { name }\end{array}$ & $\begin{array}{l}\text { Network } \\
\text { part }\end{array}$ & Short description \\
\hline $\begin{array}{l}\text { Savarese et al. } \\
\text { [57] }\end{array}$ & $\mathrm{M} 2 \mathrm{M}$ & RAN & $\begin{array}{l}\text { A frame work that allows configuring SDN-based cellular } \\
\text { network based on sensing data from M2 M devices }\end{array}$ \\
\hline $\begin{array}{l}\text { Samdanis et al. } \\
\text { [114] }\end{array}$ & D2D & RAN & $\begin{array}{l}\text { A solution to mitigate signaling overhead by introducing } \\
\text { virtual bearer and virtual gateway devices based on } \\
\text { NFV }\end{array}$ \\
\hline Chen et al. [58] & $\mathrm{M} 2 \mathrm{M}$ & RAN & Congestion control method based on SDN and openflow \\
\hline Philip et al. [60] & $\begin{array}{l}\text { Congestion } \\
\text { control }\end{array}$ & Backhaul & $\begin{array}{l}\text { Create backup paths using shared resources among } \\
\text { mobile operators in Openflow-based backhaul network }\end{array}$ \\
\hline $\begin{array}{l}\text { Gurusanthosh } \\
\text { et al. [62] }\end{array}$ & $\begin{array}{l}\text { Mobility } \\
\text { management }\end{array}$ & Backhaul & $\begin{array}{l}\text { A semi-distributed mobility anchoring in openflow-based } \\
\text { access backhaul network }\end{array}$ \\
\hline $\begin{array}{l}\text { Mahmoodi et al. } \\
\text { [63] }\end{array}$ & $\begin{array}{l}\text { Mobility } \\
\text { management }\end{array}$ & Backhaul & $\begin{array}{l}\text { Mobility management in fully realized SDN-based } \\
\text { backhaul network }\end{array}$ \\
\hline Ahmad et al. [70] & $\begin{array}{l}\text { Mobility } \\
\text { management }\end{array}$ & $\begin{array}{l}\text { Mobile } \\
\text { core }\end{array}$ & $\begin{array}{l}\text { A DMM solution for SDN-based extremely dense } \\
\text { wireless network }\end{array}$ \\
\hline $\begin{array}{l}\text { Karimzadeh et al. } \\
{[93,107,108]}\end{array}$ & $\begin{array}{l}\text { Mobility } \\
\text { management }\end{array}$ & $\begin{array}{l}\text { Mobile } \\
\text { core }\end{array}$ & $\begin{array}{l}\text { A DMM solution in virtual LTE system. The SDN } \\
\text { controller controls both DMM and EPC transportation } \\
\text { networks }\end{array}$ \\
\hline $\begin{array}{l}\text { Karimzadeh et al. } \\
{[111,133]}\end{array}$ & $\mathrm{ICN} / \mathrm{CCN}$ & $\begin{array}{l}\text { Mobile } \\
\text { core }\end{array}$ & $\begin{array}{l}\text { Integrate ICN/CCN for the support of service and VM } \\
\text { migration in the virtual LTE system }\end{array}$ \\
\hline $\begin{array}{l}\text { Dramitinos et al. } \\
\text { [112] }\end{array}$ & $\mathrm{CDN}$ & $\begin{array}{l}\text { Mobile } \\
\text { core }\end{array}$ & $\begin{array}{l}\text { Video on demand (VoD) service over SDN-based LTE } \\
\text { network }\end{array}$ \\
\hline Haw et al. [113] & $\mathrm{CCN}$ & $\begin{array}{l}\text { Mobile } \\
\text { core }\end{array}$ & $\begin{array}{l}\text { A SDN-based framework to support content delivery in } \\
\text { LTE network that consists of SDN switches with CCN } \\
\text { capable }\end{array}$ \\
\hline $\begin{array}{l}\text { Liyangage et al. } \\
\text { [117] }\end{array}$ & Security & $\begin{array}{l}\text { Mobile } \\
\text { core }\end{array}$ & $\begin{array}{l}\text { A solution to guarantee the security of the control channel } \\
\text { in a SDMN }\end{array}$ \\
\hline
\end{tabular}

network infrastructure. This architecture improves TV sessions and end-to-end QoS to satisfy the users. [123] presented IMS test-beds for future Internet research and experimentation, which are enhanced with Openflow mechanism.

Carella et al. [124] presented a design for IMS system over cloud computing system (e.g. OpenStack). The authors introduced several potential models for deploying such cloud-based IMS system and evaluated the system's performance over their OpenSDNCore architecture [125].

5.4.1.2 Service Function Chaining In this part, we consider the works [126-129] which leverage SDN into the set of middleboxes placed behind the SGi-LAN of the mobile network. StEERING [126] relied on the SDN concept to route the traffic through a series of middleboxes. StEERING allows steering different types of traffic through a sequence of desired middleboxes according to per-subscriber and per-application policies. Gember et al. $[127,128]$ introduced the concept of software defined middlebox networking to address some challenges in traditional middlebox networks, such as complex management, middlebox scaling. OpenNF [129] is a control plane architecture that allows quick and safe flows' allocation across virtual middlebox instances by combining NFV and SDN concepts. The OpenNF controller consists of a network function (NF) state manager and flow manager. The NF state manager monitors the state of virtual middle instances while the flow manager manages the forwarding rules of SDN switches. 


\section{Open Issues}

The SDN and virtualization bring many potential benefits to the mobile network and have gained a lot of attention from the research community and standardization organizations. Since the current research works are still in their infancy and in the proof-of-concept state, there are still a number of issues needed to be addressed in order to make them become reality. In the following, we will review some open issues raised in this domain.

\subsection{Backward Compatibility}

As mentioned before, there are two ways to redesign the mobile network architecture with SDN and virtualization: revolution and evolution. However, it is so difficult to move all of on-serving users into such new architectures. In the revolutionary approach, a special gateway would be required for interworking between the new revolutionary system and the legacy one. In the evolutionary approach, it is required to add the conventional interfaces to the new system in order to co-work with the legacy system. Therefore, SDVMN needs to find a flexible and effective design strategy to be compatible with the existing mobile network.

\subsection{Unified Control Plane}

Since many works have introduced SDN into various levels of mobile networks, their design of the controller's functionality and the controlling interface are different. In order to achieve a complete solution for SDN and virtualization in the mobile network, it is important to have standardized interfaces and unified controller platforms.

\subsection{Deployment Model}

The deployment choice for the network function should take into account various approaches, such as full virtualization or partial virtualization, and full centralization or partial centralization [76]. In addition, there are two deployment choices for the control plane: centralized and distributed. For example, the use of physically distributed multiple controllers can mitigate the single point of failure and increase the scalability. Therefore, the key issue is to find a trade-off among various deployment models with careful evaluation.

\subsection{Mobility Management}

With the adoption of SDN and virtualization in the mobile network, we will witness the arrival of virtual network operators. In addition, with the dense deployment of multiple wireless technologies, how to handle the seamless connection among these virtual network operators and heterogeneous access networks is an open issue that must be addressed. Besides, mechanisms supporting fast handover are necessary to achieve different requirements in the QoS and QoE in such new dense and heterogeneous architecture.

\subsection{Security}

Security problems in the SDN and virtualization based mobile network come from the vulnerability of the controller and the lack of secure mechanisms in the control channel 
[117]. Several solutions that deal with these problems are well-known in the wired SDN/ Openflow network $[130,131]$. However, these solutions need to be further studied for such new SDN-based wireless mobile networks.

\subsection{Supporting New Network Paradigms}

With the support of SDN and virtualization, the mobile cellular network promises to bring new benefits for upcoming applications and services or new network paradigms, such as IoT applications [132], D2D [56] communication, or ICN [110]. However, there is few research works related to these new paradigms in SDVMN architecture. Therefore, this topic opens many potential future research opportunities to the community.

\section{Conclusion}

Without solving the fundamental issues, such as proprietary hardware devices, complicated management, and inefficient resource utilization regarding the inherent design of mobile networks, it is very difficult for network operators to cope with the challenges exposed by the mobile Internet era. SDN and virtualization feature the centralized control plane, programmable network, software-based network functions, and physical infrastructure sharing. These are two complementary and promising technologies that provide solutions to major issues of mobile networks as well as facilitate many of their aspects. The adoption of SDN and virtualization in the mobile network has attracted many research works in academia and industry. In this paper, we surveyed comprehensively the latest approaches for SDN and virtualization-based mobile networks by covering a wide range of up-to-date research works related to this topic. We first made a general architecture for SDN and virtualization in the mobile network, and describe in detail benefits that these two technologies bring in each level of the cellular network structure. Next, we proposed a hierarchical taxonomy based on the various levels of the mobile network structure in order to classify current research works. Through this taxonomy, we can understand what issues raised in the current design of the mobile network and promising solutions for each issue. By using this taxonomy, we looked deeply into each research work and focused on changes regarding the architecture and protocol operation when adopting the SDN and virtualization in each level of carrier networks. Then, we showed a list of use cases and applications that can take advantages of SDN and virtualization. Last, we discussed open issues, such as compatibility, deployment model, and unified control plane that need to be addressed in order to implement the SDN and virtualization-based mobile network in reality. In summary, SDN and virtualization will be two key enable technologies for the evolution of future mobile networks. However, among current proposals, which will be the most suitable and efficient solution for SDN and virtualization-based mobile network is still an open issue. This choice requires the careful consideration of network operators and research communities based on economic and technical benefits.

Acknowledgments This work was supported by Institute for Information \& communications Technology Promotion (IITP) grant funded by the Korea government (MSIP) (No. B0190-15-2012, Global SDN/NFV OpenSource Software Core Module/Function Development). 
Open Access This article is distributed under the terms of the Creative Commons Attribution 4.0 International License (http://creativecommons.org/licenses/by/4.0/), which permits unrestricted use, distribution, and reproduction in any medium, provided you give appropriate credit to the original author(s) and the source, provide a link to the Creative Commons license, and indicate if changes were made.

\section{References}

1. Cisco. (2014). Cisco visual networking index: Forecast and methodology, 2013-2018. http://www. cisco.com/c/en/us/solutions/collateral/service-provider/ip-ngn-ip-next-generation-network/white_paper_ c11-481360.pdf. Accessed August 26, 2014.

2. Open Networking Foundation (ONF). (2013). Openflow-enabled mobile and wireless networks. ONF solution brief. https://www.opennetworking.org/images/stories/downloads/sdn-resources/solutionbriefs/sb-wireless-mobile.pdf. Accessed March 2013.

3. ETSI. (2013). Network function virtualization (NFV): Use cases. ETSI group specification. http:// www.etsi.org/deliver/etsi_gs/NFV/001_099/001/01.01.01_60/gs_NFV001v010101p.pdf. Accessed August 2014.

4. Open Networking Foundation (ONF). (2012). Software-defined networking: The new norm for networks. ONF white paper. https://www.opennetworking.org/images/stories/downloads/sdn-resources/ white-papers/wp-sdn-newnorm.pdf. Accessed August 2014.

5. McKeown, N., Anderson, T., Balakrishnan, H., Parulkar, G., Peterson, L., Rexford, J., et al. (2008). Openflow: Enanbling innovations in campus networks. ACM SIGCOMM Computer Communication Review (CCR), 38(2), 69-74.

6. Open Networking Foundation (ONF). (2013). Openflow specification version 1.4.0. ONF specifications. https://www.opennetworking.org/images/stories/downloads/sdn-resources/onf-specifications/ openflow/openflow-spec-v1.4.0.pdf. Accessed August 2014.

7. Doria, A., Salim, J. H., Haas, R., Khosravi, H., Wang, W., \& Dong, L., et al. (2010). Forwarding and control elelment separation (ForCES) protocol specification. IEFT RFC 5810. https://tools.ietf.org/ html/rfc5810. Accessed May 2015.

8. Vasseur, J. P., \& Le Roux, J. L. (2009). Path computation elelement (PCE) communication protocol (PCEP). IETF RFC 5440. https://tools.ietf.org/html/rfc5440. Accessed May 2015.

9. Chowdhury, N. M. M. K., \& Boutaba, R. (2010). A survey of network virtualization. Computer Networks, 54(5), 862-876.

10. ETSI. (2013). Network functions virtualization white paper. http://portal.etsi.org/nfv/nfv_white_ paper2.pdf. Accessed February 2014.

11. GPPP. (2010). The 5G infrastructure public-private partnership http://5g-ppp.eu/. Accessed May 2014.

12. METIS project. (2012). Mobile and wireless communications enablers for the twenty-twenty information society. http://www.metis2020.com/. Accessed May 2014.

13. iJOIN project. (2013). Interworking and joint design of an open access and backhaul network architecture for small cells based on cloud networks. http://www.ict-ijoin.eu/. Accessed May 2014.

14. Mobile Cloud Networking project. (2013). http://mobile-cloud-networking.eu/. Accessed May 2014.

15. CROWD project. (2013). Connectivity management for energy optimised wireless dense networks. http://www.ict-crowd.eu/. Accessed May 2014.

16. Tomovic, S., Pejanovic-Djurisic, M., \& Radusinovic, I. (2014). SDN based mobile networks: Concepts and benefits. Wireless Personal Communications, 78(3), 1629-1644.

17. Yang, M., Li, Y., Jin, D., Zeng, L., Wu, X., \& Vasilakos, A. V. (2014). Software defined and virtualized future mobile and wireless networks: A survey. Mobile Networks and Applications. doi:10. 1007/s11036-014-0533-8.

18. 3GPP. (2012). General packet radio service (gprs) enhancements for evolved universal terrestrial radio access network (e-utran) access. Technical specification (TS 23.401 v10.8.0). http://www.3gpp.org/ ftp/Specs/archive/23_series/23.401/23401-a80.zip. Accessed May 2014.

19. Open Networking Foundation (ONF). (2013). https://www.opennetworking.org. Accessed July 2013.

20. Jarraya, Y., Madi, T., \& Debbabi, M. (2014). A survey and a layered taxonomy of software-defined networking. IEEE Communications Survey and Tutorials, 16(4), 1955-1980.

21. Kreutz, D., Ramos, F., Verissimo, P., Rothenberg, C. E., Azodolmolky, S., \& Uhlig, S. (2014). Software-defined networking: A comprehensive survey. http://arxiv.org/pdf/1406.0440v1.pdf. Accessed 15 June 2014. 
22. Nunes, B., Mendonca, M., Nguyen, X. N., Obraczka, K., \& Turletti, T. (2014). A survey of softwaredefined networking: Past, present, and future of programmable networks. IEEE Communications Survey and Tutorials, 16(3), 1617-1634.

23. Floodlight project. (2013). http://www.projectfloodlight.org/. Accessed May 2013.

24. OpenDaylight project. (2013). OpenDaylight technical overview. http://www.opendaylight.org/ project/technical-overview. Accessed May 2013.

25. OpenDaylight Project. (2014). OpenDaylight sdni application. https://wiki.opendaylight.org/view/ Project_Proposals:ODL-SDNi_App. Accessed August 2014.

26. Sezer, S., Scott-Hayward, S., Chouhan, P. K., Fraser, B., Lake, D., Finnegan, J., et al. (2013). Are we ready for sdn? Implementation challenges for software-defined networks. IEEE Communications Magazine, 51(7), 36-43.

27. PlannetLab project. (2013). http://www.planet-lab.org/. Accessed August 2014.

28. Achemlal, M., Almeida, S., Gorg, C., \& Bourguiba, M. et al. (2009). D-3.2.0 virtualization approach: Concept. The FP7 4WARD project, technical report.

29. GENI. (2013). Global environment for network innovations. http://www.geni.net/. Accessed July 2014.

30. Bavier, A., Feamster, N., Huang, M., Peterson, L., \& Rexford, J. (2006). In vini veritas: Realistic and controlled network experimentation. ACM SIGCOMM Computer Communication Reivew (CCR), 36(4), 3-14.

31. Sherwood, R., Chan, M., Covington, A., et al. (2010). Carving research slices out of your production networks with openflow. ACM SIGCOMM Computer Communication Reivew (CCR), 38(3), 105-110.

32. Al-Shabibi, A., Leenheer, M. D., Koshibe, A., Parulkar, G., Snow, B., Gerola, M., \& Salvadori, E. (2014). OpenVirteX: Make your virtual sdns programmable. In the 3rd ACM SIGCOMM workshop on hot topics in software defined networking (HotSDN) (pp. 25-30).

33. European Telecommunications Standards Institute (ETSI). (2012). http://www.etsi.org/technologiesclusters/technologies/nfv. Accessed February 2014.

34. ETSI. (2012). Network functions virtualisation: An introduction, benefits, enablers, challenges and call for action. http://portal.etsi.org/NFV/NFV_White_Paper.pdf. Accessed February 2014.

35. ETSI. (2013). Network functions virtualisation: Architectural framework. Group specification. http:// www.etsi.org/deliver/etsi_gs/NFV/001_099/002/01.01.01_60/gs_NFV002v010101p.pdf. Accessed August 2014.

36. John, W., Pentikousis, K., Agapiou, G., Jacob, E., Kind, M., \& Manzalini, A., et al. (2013). Research directions in network service chaining. In IEEE workshop on software defined network for future networks and services (SDN4FNS) (pp. 1-7).

37. ONF. (2013). SDN architecture overview. https://www.opennetworking.org/images/stories/downloads/ sdn-resources/technical-reports/SDN-architecture-overview-1.0.pdf. Accessed May 2014.

38. ONF. (2013). Northbound interfaces working group charter. https://www.opennetworking.org/images/ stories/downloads/working-groups/charter-nbi.pdf. Accessed May 2014.

39. Liang, C., \& Yu, F. R. (2014). Wireless network virtualization: A survey, some research issues and challenges. IEEE Communications Survey and Tutorials, 17(1), 358-380.

40. Chen, C. (2011). C-RAN: The road towards green ran. China Mobile Research Institute. http://labs. chinamobile.com/cran/wp-content/uploads/CRAN_white_paper_v2_5_EN.pdf. Accessed May 2014.

41. Hadzialic, M., Dosenovic, B., Dzaferagic, M., \& Musovic J. (2013). Cloud-RAN: Innovative radio access network architecture. In The 55th ELMAR international symposium (pp. 115-120).

42. Checko, A., Christiansen, H. L., Yan, Y., Scolari, L., Kardaras, G., Berger, M. S., \& Dittmann, L. (2014). Cloud ran for mobile networks-A technology overview. IEEE Communications Survey and Tutorials, 17(1), 405-426.

43. Liu, J., Zhao, T., Zhou, S., Cheng, Y., \& Niu, Z. (2014). CONCERT: A cloud-based architecture for next-generation cellular systems. IEEE Wireless Communications, 21(6), 14-22.

44. Rost, P., Bernardos, C. J., Domenico, A. D., Girolamo, M. D., Lalam, M., Maeder, A., et al. (2014). Cloud technoligies for flexbile $5 \mathrm{G}$ radio access network. IEEE Communication Magazine, 52(5), 68-76.

45. Sabella, D., Rost, P., Sheng, Y., Pateromichelakis, E., Salim, U., \& Ouhamou, P. G., et al. (2013). RAN as a service: Challenges of designing a flexible ran architecture in a cloud-based heterogenerous mobile network. In IEEE future network and mobile summit (futurenetworksummit) (pp. 1-8).

46. Yang, Mao, Li, Y., Jin, D., Su, L., Ma, S., \& Zeng, L. (2013). OpenRAN: A software-defined ran architecture via virtualization. ACM SIGCOMM Computer Communication Reivew (CCR), 43(4), 549-550.

47. Gudipati, A., Perry, D., Li, L. E., \& Katti, S. (2013). SoftRAN: Software defined radio access network. In ACM SIGCOMM 2nd workshop on hot topics in software defined networking (HotSDN) (pp. 25-30). 
48. Wu, W., Li, L. E., Panda, A., \& Shenker, S. (2014). PRAN: Programmable radio access networks. In The 13th ACM workshop on hot topics in networks (HotNets-XIII) (pp. 1-7).

49. Riggio, R., Goratti, L., Fedrizzi, R., \& Rasheed, T. (2014). V-Cell: Going beyond the cell abstraction in $5 \mathrm{G}$ mobile networks. In IEEE network operations and management symposium (NOMS), (pp.1-5).

50. Gudipati, A., Li, L. E., \& Katti, S. (2014). RadioVisor: A slicing plane for radio access networks. In ACM SIGCOMM 3rd workshop on hot topics in software defined networking (HotSDN) (pp. 237-238).

51. Spapis, P., Chatzikokolakis, K., Alonistioti, N., \& Kaloxylos, A.(2014). Using sdn as a key enabler for co-primary spectrum sharing. In 5th IEEE international conference oon information, intelligence, systems and applications (IISA) (pp. 366-371).

52. Costanzo, S., Xenakis, D., Passas, N., \& Merakos, L. (2014). OpeNB: A framework for virtualizing base stations in lte networks. In IEEE international conference on communications (ICC) (pp. 3148-3153).

53. Zaki, Y., Zhao, L., Goerg, C., \& Giel, A. T. (2011). LTE mobile network virtualization. Mobile Networks and Applications, 16(4), 424-432.

54. Amani, M., Mahmoodi, T., Tatipamula, M., \& Aghvami, H. (2014). Programmable policies for data offloading in lte network. In IEEE international conference on communications (ICC) (pp. 3154-3159).

55. Kim, J., Lee, J., Kim, J., \& Yun, J. (2014). M2M service platforms: Survey, issues, and enabling technologies. IEEE Communications Survey and Tutorials, 16(1), 67-76.

56. Asadi, A., Wang, Q., \& Mancuso, V. (2014). A survey on device-to-device communication in cellular networks. IEEE Communications Survey and Tutorials, 16(4), 1801-1819.

57. Savarese, G., Vaser, M., \& Ruggieri, M. (2013). A software defined networking-based context-aware framework combining $4 \mathrm{G}$ cellular networks with $\mathrm{m} 2 \mathrm{~m}$. In The 16th IEEE international symposium on wireless personal multimedia communications (WPMC) (pp. 1-6).

58. Chen, J. L., Hsieh, H. C., \& Larosa, Y. T. (2013). Congestion control optimization of $\mathrm{m} 2 \mathrm{~m}$ in 1 te networks. In The 15th international conference on advanced communications technology (ICACT) (pp. 823-827).

59. Philip, V. D., Gourhant, Y., \& Zeghlache, D. (2011). Mobile network sharing using openflow. In CHANGE\&OFELIA summer workshop. http://changeofelia.info.ucl.ac.be/pmwiki/uploads/Summer School/Program/poster_018.pdf. Accessed May 2014.

60. Philip, V. D., Gourhant, Y., \& Zeghlache, D. (2012). Demystifying link congestion in 4G-lte backhaul using openflow. In The 5th IEEE international conference on new technologies, mobility and security (NTMS) (pp. 1-8).

61. Philip, V. D., Gourhant, Y., \& Zeghlache, D. (2011). Preliminary analysis of 4G-lte mobile network sharing for improving resiliency and operator differentiation. E-Technologies and networks for Development, 171, 73-93.

62. Gurusanthosh, P., Rostami, A., \& Manivasakan, R. (2013). SDMA: A semi-distributed mobility anchoring in lte networks. In IEEE international conference on selected topics in mobile and wireless networking (MoWNeT) (pp. 133-139).

63. Mahmoodi, T., \& Seetharaman, S. (2014). Traffic jam: Handling the increasing volume of mobile data traffic. IEEE Vehicular Technology Magazine, 9(3), 56-62.

64. Matsushima, S., \& Wakikawa, R. (2014). Stateless user-plane architecture for virtualized epc (vepc). http://tools.ietf.org/html/draft-matsushima-stateless-uplane-vepc-03. Accessed August 2014.

65. Jin, X., Li, L. E., Vanbever, L., \& Rexford, J. (2013). Softcell: Scalable and exible cellular core network architecture. In ACM conference on emerging networking experiments and technologies (CoNEXT) (pp. 163-174).

66. Li, L. E., Mao, Z. M., \& Rexford, J. (2012). Toward software-defined cellular networks. In The 1st European workshop on software defined networking (EWSDN) (pp. 7-12).

67. Moradi, M., Li, L. E., \& Mao, Z. M. (2014). SoftMoW: A dynamic and scalable software defined architecture for cellular wans. In ACM SIGCOMM 3rd workshop on hot topics in software defined networking (HotSDN) (pp. 21-22).

68. Moradi, M., Li, L. E., \& Mao, Z. M. (2014). SoftMoW: Recursive and reconfigurable cellular wan architecture. In 10th ACM international conference on emergin networking experiments and technologies (CoNEXT) (pp. 377-390).

69. Bernardos, C. J., Oliva, A. D. L., Serrano, P., Banchs, A., Contreras, L. M., Jin, H., \& Zuniga, J. C. (2014). An architecture for software defined wireless networking. IEEE Wireless Communications, 21(3), 52-61.

70. Ahmad, H. A., Cicconetti, C., Oliva, A. D. L, Mancuso, V., Sama, M. R., Seite, P., \& Shanmugalingam, S. (2013). An sdn-based network architecture for extremely dense wireless networks. In IEEE workshop on software defined network for future networks and services (SDN4FNS) (pp. 1-7). 
71. Ahmad, H. A., Cicconetti, C., Oliva, A. D. L, Gupta, R., Mancuso, V., Roullet, L., \& Sciancalepore, V. (2013). CROWD: An sdn approach for densenets. In The 2nd European workshop on software defined networking (EWSDN) (pp. 25-31).

72. Guerzoni, R., Trivisonno, R., \& Soldani, D. (2014). SDN-based architecture and procedures for 5G networks. In 1st IEEE international conference on $5 G$ for ubiquitous connectivity $(5 G U)$ (pp. 1-6).

73. Trivisonno, R., Guerzoni, R., Vaishnavi, I., \& Soldani, D. (2015). SDN-based 5G mobile networks: Architecture, functions, procedures and backward compatibility. Transactions on Emerging Telecommunications Technologies, 26(1), 82-92.

74. Shanmugalingnam, S., \& Bertin, P. (2014). Programmable mobile core network. In 19th IEEE symposium on computers and communications (ISCC) (pp. 1-7).

75. Pentikousis, K., Wang, Y., \& Hu, W. (2013). MobileFlow: Towards software-defined mobile networks. IEEE Communications Magazine, 51(7), 44-53.

76. Basta, A., Kellerer, W., Hoffmann, M., Hoffmann, K., \& Schmidt, E. D. (2013). A virtual sdn-enabled lte epc architecture: A case study for s-/p-gateways functions. In IEEE workshop on software defined network for future networks and services (SDN4FNS) (pp. 1-7).

77. Basta, A., Kellerer, W., Hoffmann, M., Morper, H. J., \& Hoffmann, K. (2014). Applying nfv and sdn to lte mobile core gateways; the functions placement problem. In ACM workshop on all things cellular: Operators, applications, and challenges (AllThingsCellular) (pp. 33-38).

78. Basta, A., Blenk, A. Hoffmann, M., Morper, H. J., Hoffmann, K., \& Kellerer, W. (2014). Sdn and nfv dynamic operation of lte epc gatewaysfor time-varying traffic patterns. In The 6th international conference on mobile networks and management (MONAMI). http://www.lkn.ei.tum.de/forschung/ publikationen/dateien/Basta2014SDNandNFVDynamic.pdf. Accessed August 2014.

79. MEVICO project. (2012). D2.2: Architectural epc extensions for supporting heterogenerous mobility schemes. Technical report. http://www.mevico.org/D22.pdf. Accessed May 2014.

80. Heinonen, J., Partti, T., Kallio, M., Lappanlainen, K., Flinck, H., \& Hillo, J. (2014). Dynamic tunnel switching for sdn-based cellular core networks. In ACM workshop on all things cellular: Operators, applications, and challenges (AllThingsCellular) (pp. 27-32).

81. Kempf, J., Johansson, B., Pettersson, S., \& Luning, H. (2012). Moving the evolved packet core to the cloud. In The 8th IEEE international conference on wireless and mobile computing, networking and communications (WiMob) (pp. 784-791).

82. Kempf, J., Johansson, B. E., Pettersson, S., Nilsson, T. K., \& Luning, H. (2012). Implementing epc in a cloud computer with openflow data plane. US Patent (US 2012/0300615A1).

83. Kempf, J., Zavarch, N. B., Nilsson, T. K., Johansson, B. E., Pettersson, S. R., \& Luning, H. (2013). Implementing a $3 \mathrm{G}$ packet core in a cloud computer with openflow data and control planes. US Patent (US 2013/0054761A1).

84. Mueller, J., Chen, Y., Reichel, B., Vlad, V., \& Magedanz, T. (2014). Design and implementation of a carrier grade software defined telecommunication switch and controller. In IEEE network operations and management symposium (NOMS) (pp.1-7).

85. Said, S. B. H, Sama, M. R., Guillouard, K., Suciu, L., Simon, G., Lagrange, X., \& Bonnin, J. M. (2013). New control plane in $3 \mathrm{gpp}$ lte/epc architecture for on-demand connectivity service. In The 2nd IEEE international conference on cloud networking (CloudNet) (pp. 205-209).

86. Sama, M. R., Said, S. B. H., Guillouard, K., \& Suciu, L. (2014). Enabling network programmability in lte/epc architecture using openflow. In The 12th IEEE international symposium on modeling and optimization in mobile, ad hoc, and wireless networks (WiOpt) (pp. 395-402).

87. Nguyen, V. G., \& Kim, Y. H. (2014). Signaling load analysis in openflow-enabled lte/epc architecture. In The 5th IEEE international conference on ICT convergence (ICTC) (pp. 734-735).

88. Nguyen, V. G., \& Kim, Y. H. (2015). Proposal and evaluation of sdn-based mobile packet core networks. EURASIP Journal on Wireless Communications and Networking, 2015(172), 1-18.

89. Gomez, K., Rasheed, T., Reynaud, L., \& Goratti, L. (2014). FME: A flexible management entity for virtualizing lte evolved packet core. In IEEE network operations and management symposium (NOMS) (pp.1-4).

90. Gomez, K., Goratti, L., Rasheed, T., \& Reynaud, L. (2014). Enabling disaster resilient 4 g mobile communication networks. IEEE Communications Magazine, 52(12), 66-73.

91. Nagaraj, K., \& Katti, S. (2014). ProCel: Smart traffic handling for a scalable software epc. In $A C M$ SIGCOMM 3rd workshop on hot topics in software defined networking (HotSDN) (pp. 43-48).

92. Kunz, A., Palanimuthu, S., Song, J., \& Kolbe, H. J. (2013). MC-SDN: Introducing openflow in the $3 \mathrm{gpp}$ evolved packet core. IEEE Communications Society E-Letter, 8(3), 40-42.

93. Karimzadeh, M., Valtulina, L., \& Karagiannis, G. (2014). Applying sdn/openflow in virtualized lte to support distributed mobility management $(\mathrm{dmm})$. In The 4th international conference on cloud computing and services science (CLOSER) (pp.1-6). 
94. Karagiannis, G., Jamakovic, A., Admonds, A., Parada, C., Metsch, T., \& Pichon, D., et al. (2014). Mobile cloud networking: Virtualisation of cellular networks. In The 21st IEEE international conference on telecommunications (ICT) (pp. 410-415).

95. Hawilo, H., Shami, A., Mirahmadi, M., \& Asal, R. (2014). NFV: State of the art, challenges and implementation in next generation mobile networks (vepc). IEEE Network Magazine, 28(6), 18-26.

96. Yousaf, F. Z., Lessmann, J., Loureiro, P., \& Schmid, S. (2013). SoftEPC-Dynamic instantiation of mobile core network entities for efficient resource utilization. In IEEE international conference on communications (ICC) (pp. 3602-3606).

97. Hampel, G., Steiner, M., \& Bu, T. (2013). Applying software-defined networking to the telecom domain. In IEEE conference on computer communications workshops (INFOCOM WKSHPS) (pp.133-138).

98. Nguyen, V. G., \& Kim, Y. H. (2014). Slicing the next mobile packet core network. In The 11th IEEE international symposium on wireless communication systems (ISWCS) (pp. 901-904).

99. Ghazisaeedi, E., \& Tafazolli, R. (2013). Mobile core traffic balancing by openflow switching system. In IEEE globecom workshops (GC Wkshps) (pp. 824-829).

100. Cho, J., Nguyen, B., Banerjee, A., Ricci, R., Merwe, J. V. D., \& Webb, K. (2014). SMORE: Softwaredefined networking mobile offloading architecture. In ACM workshop on all things cellular: Operators, applications, and challenges (AllThingsCellular) (pp. 21-26).

101. Banerjee, A., Chen, X., Erman, J., Gopaklakrishnan, V., Lee, S., \& Merwe, J. V. D. (2013). MOCA: A lightweight mobile cloud offloading architeture. In The 8th ACM international workshop on mobility in the evolving internet architecture (MobiArch) (pp. 11-16).

102. Ding, A. Y., Crowcroft, J., \& Tarkoma, S. (2014). SoftOffload: A programmable approach toward collaborative mobile traffic offloading. In the 12th annual international conference on mobile systems, applications, and services (MobiSys) (pp. 368-368).

103. Donatini, L., Garroppo, G., Giordano, S., Foddis, Gianluca, \& Topazzi, S. (2014). Advances in lte network monitoring: A step towards an sdn solution. In The 17th IEEE mediterranean electronical conference (MELECON) (pp. 339-343).

104. Bigwitch networks. (2014). Nex-generation monitoring fabrics for mobile networks. White paper. http://www.bigswitch.com/solutions/next-generation-monitoring-fabrics-for-mobile-lte-core-networks. Accessed August 2014.

105. Chan, H. A., Yokota, H., Xie, J., Seite, P., \& Liu, D. (2011). Distributed and dynamic mobility management in mobile internet: Current approaches and issues. Journal of Communications, 6(1), $4-15$.

106. CROWD project. (2013). D4.1: Initial specification of connectivity management concepts and architecture. Technical report. http://www.ict-crowd.eu/downloads/deliverable/CROWD_D4.1.pdf. Accessed May 2014.

107. Karimzadeh, M., Sperotto, A., \& Pras, A. (2014). Software defined networking to improve mobility management performance. In Monitoring and securing virtualized networks and services, lecture notes in computer science (LNCS) (pp. 118-122). Berlin: Springer.

108. Valtulina, L., Karimzadeh, M., Karagiannis, G., Heijenk, G., \& Pras, A. (2014). Performance evaluation of a sdn/openflow-baed distributed mobility management (dmm) approach in virtualized lte systems. In IEEE GLOBECOM workshop on cloud computing systems, networks, and applications (CCSNA) (pp. 18-23).

109. Kuklinski, S., Li, Y., \& Dinh, K. T. (2014). Handover management in sdn-based mobile networks. In IEEE GLOBECOM workshop on management of emerging networks and services (MENS) (pp. 1-7).

110. Ahlgren, B., Dannewitz, C., Imbrenda, C., Kutscher, D., \& Ohlman, B. (2012). A survey of information-centric networking. IEEE Communication Magazine, 50(7), 26-36.

111. Karimzadeh, M., Satria, T., \& Karagiannis, G. (2014). Utilizing icn/ccn for service and vm migration support in virtualized lte systems. In The 4th international conference on cloud computing and services science (CLOSER) (pp.1-6).

112. Dramitios, M., Kantor, M., Zhang, N., \& Requena, J. C. (2013). Video delivery over next genration cellular networks. In The 9th international conference on network and service management (CNSM) (pp. 386-393).

113. Haw, R., Hong, C. S., \& Lee, S. (2014). An efficient content delivery framework for sdn based lte network. In The 8th ACM international conference on ubiquitous information management and communication (UCUIMC) (p. 71).

114. Samdanis, K., Kunz, A., Hossain, I. H., \& Taleb, T. (2013). Virtual bearer management for efficient mtc radio and backhaul sharing in lte networks. In The 24th IEEE international symposium on personal, indoor and mobile radio communications (PIMRC) (pp. 2780-2785). 
115. Yazici, V., Kozat, U. C., \& Sunay, M. O. (2014). A new control plane for 5G network architecture with a case study on unified handoff, mobility, and routing management. IEEE Communications Magazine, 52(1), 76-85.

116. Taleb, T., Ksentini, A., \& Kobbane, A. (2014). Lightweight mobile core networks for machine type communications. IEEE Access, 2, 1128-1137.

117. Liyanage, M., Ylianttila, M., \& Gurtove A. (2014). Securing the control channel of software-defined mobile networks. In The 1st IEEE WoWMoM workshop on software defined networking architecture and applications (SDN-AA).

118. Lu, F., Pan, H., Lei, X., Liao, X., \& Jin, H. (2013). A virtualization-based cloud infrastructure for ims core network. In The 5th IEEE international conference on cloud computing technology and science (CloudCom) (pp. 25-32).

119. Ito, M., Nakauchi, K., Shoji, Y., \& Kitatsuji, Y. (2014). Mechanism of reducing signaling processing load in epc/ims using service-specific network virtualization. In The 6th IEEE international conference on new technologies, mobility and security (NTMS) (pp. 1-5).

120. Ito, M., Nakauchi, K., Shoji, Y., Nishinaga, N., \& Kitatsuji, Y. (2014). Service-specific network virtualization to reduce signaling processing loads in epc/ims. IEEE Access, 2, 1076-1084.

121. Ito, M., Komorita, S., Kitatsuji, Y., \& Yokota, H. (2013). Openflow-based routing mechanism for call session state migration in the ims. In The proceeding of 7 th international conference on recent advances in computer engineering series (WSEAS).

122. Truong, T. H., Nguyen, T. H., Mueller, J., \& Magedanz, T. (2013). QoE-aware resource provisioning and adaptation in ims-based iptv using openflow. In The 19th IEEE workshop on local and metropolitan area networks (LANMAN) (pp. 1-3).

123. Tranoris, C., Denazis, S., Mouratidis, N., Dowling, P., \& Tynan, J. (2013). Integrating openflow in ims networks and enabling for future internet research and experimentation. In The future internet, lecture notes in computer science (LNCS) (pp. 77-88). Berlin: Springer.

124. Carella, G., Corici, M., Crosta, P., Comi, P., Bohnert, T. M., Corici, A. A., Vingarzan, D., \& Magedanz, T. (2014). Cloudified ip multimedia subsystem (ims) for network function virtualization (nfv)-based architectures. In IEEE international conference on computers and communcications (ISCC), (pp. 1-6).

125. Fraunhofer FOKUS. OpenSDNCore-Reasearch and testbed for the carrier-grade nfv/sdn environment. http://www.opensdncore.org/. Accessed October 2014.

126. Zhang, Y., Beheshti, N., Beliveau, L, Lefebvre, G., Manghirmalani, R., \& Mishra, R., et al. (2013). StEERING: A software-defined networking for inline service chaining. In The 21st IEEE international conference on network protocols (ICNP) (pp. 1-10).

127. Gember, A., Prabhu, P., Ghadiyali, Z., \& Akella, A. (2012). Toward software-defined middlebox networking. In The 11th ACM workshop on hot topics in networks (HotNest) (pp. 7-12).

128. Gember, A., Grandl, R., Khalid, J., \& Akella, A. (2013). Design and implementation of a framework for software-defined middlebox networking. ACM SIGCOMM Computer Communication Review (CCR), 43(4), 467-468.

129. Gember, A., Viswanathan, R., Prakash, C., Grandl, R., Khalid, J., Das, S., \& Akella, A. (2014). OpenNF: Enabling inovation in network function control. ACM SIGCOMM Computer Communication Review (CCR), 44(4), 163-174.

130. Hayward, S. S., O'Callaghan, G., \& Sezer, S. (2013). SDN security: A survey. In IEEE workshop on software defined network for future networks and services (SDN4FNS) (pp. 1-7).

131. Hegde, N., \& Hu, F. (2014). Security issues in sdn/openflow. In H. Fei (Ed.), Network innovation through openflow and sdn (pp. 415-434). New York: CRC Press.

132. Li, S., Xu, L. D., \& Zhao, S. (2014). The internet of things: A survey. Information Systems Frontiers, $17(2), 243-259$.

133. Satria, T. A., Karimzadeh, M., \& Karagiannis, G. (2014). Performance evaluation of icn/ccn based service migration approach in virtualized lte systems. In The 3rd IEEE international conference on cloud networking (CloudNet) (pp. 461-467). 

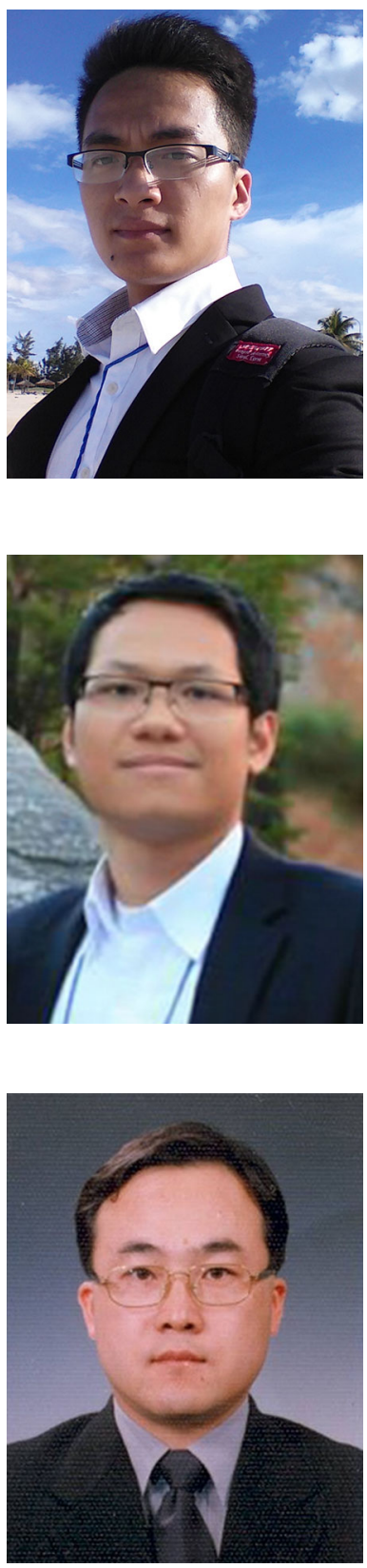

Van-Giang Nguyen graduated in Electronics and Telecommunication Engineering in 2012 at Hanoi University of Science and Technology, Vietnam and the Master Degree in 2014 from the Department of Information and Telecommunication Engineering, Soongsil University in Korea. He is currently research assistant in Soongsil University. His main research interests are SDN (software defined networking), Openflow, future mobile network architecture, DMM (distributed mobility management), NFV (network function virtualization), SDN and virtualization in mobile network architecture, 5G mobile packet core network, SFC (service function chaining), ICN (information centric networking). He is a member of IEEE SDN.

Truong-Xuan Do received the B.E. degree in Electronics and Telecommunication Engineering from the Hanoi University of Science and Technology, Vietnam, in 2011 and the M.S degree in Information and Telecommunication Engineering from the Soongsil University, Korea in 2013.He is currently a Ph.D. candidate at Department of Information and Telecommunication Engineering, Soongsil University, Korea. His current research interests include mobility management and multicast service in next generation wireless network, DMM (distributed mobility management), SDN (software defined networking) in mobile network, ICN (information centric networking), and network virtualization.

YoungHan Kim received his B. S. degree in electronics engineering from Seoul National University, Seoul, Korea, in 1984, and the M. S. and $\mathrm{Ph} . \mathrm{D}$. degrees in electrical engineering from Korea Advanced Institute of Science and Technology, Seoul, in 1986 and 1990 respectively. From 1987 to 1994 he was a senior member of the research staff in the Digicom Institute of Telemetics, Seoul, Korea, where he did research on data and computer communication systems. Since September 1994 he has been with Soongsil university, where he is a professor of school of electronic engineering. He is a director of Ubiquitous Network Research Center and a director of ConvergenceITRC (Convergence Information Technology Research Center) project supported by MSIP(Ministry of Science, ICT and Future Planning), Korea. His research interests include wireless networking and future networking, SDN (Software Defined Networking), ICN (Information Centric Networking) and sensor networking. He is a member of IEICE and IEEE. 